SUPPORTING INFORMATION (51 PAGES)

OCTOBER 25, 2020

\title{
Intrastrand Photolesion Formation in Thiosubstituted DNA: A Case Study Including Single-Reference and Multireference Methods
}

Eva Vos, ${ }^{1}$ Thais Scott, ${ }^{2}$ Jesús González-Vázquez, ${ }^{* 1,3}$ Inés Corral, ${ }^{* 1,3}$

Donald G. Truhlar, ${ }^{* 4}$ and Laura Gagliardi*2

${ }^{1}$ Departamento de Química, Módulo 13, Universidad Autónoma de Madrid, 28049 Madrid, Spain

${ }^{2}$ Department of Chemistry, Pritzker School of Molecular Engineering, James Franck Institute, Chicago Center for Theoretical Chemistry, The University of Chicago, 5735 S Ellis Ave, Chicago, IL 60637, United States

${ }^{3}$ Institute of Advanced Chemical Sciences (IadChem), Universidad Autońoma de Madrid, Campus de Excelencia UAM-CSIC, Cantoblanco, 28049 Madrid, Spain

${ }^{4}$ Department of Chemistry, Chemical Theory Center, and Minnesota Supercomputing Institute, University of Minnesota, 207 Pleasant Street SE, Minneapolis, Minnesota 55455-0431, United States

\section{TABLE OF CONTENTS}

\section{Results with the cc-pVDZ basis set in multiconfigurational calculations}

Absolute energies of the $S_{0}$ stationary points

Absolute energies of the $T_{1}$ stationary points

Absolute energies of ground and excited states along the $\mathrm{S}_{0}$ mechanisms

Absolute energies of ground-state along the $\mathrm{T}_{1}$ mechanisms

Orbitals in the active space used in the multireference

Multireference $\mathrm{M}$ diagnostic for the lowest lying singlet and triplet electronic states along the $\mathrm{S}_{0}$ and $\mathrm{T}_{1}$ mechanisms 
Effect of polarization functions in the basis set

S-12

Results with the ANO-RCC-VDZ basis set in multiconfigurational calculations

$\begin{array}{lr}\text { Absolute energies of the } S_{0} \text { stationary points } & \text { S-14 }\end{array}$

$\begin{array}{ll}\text { Absolute energies of the } \mathrm{T}_{1} \text { stationary points } & \mathrm{S}-14\end{array}$

Absolute energies of ground and excited states along the $S_{0}$ mechanisms $\quad$ S-15

$\begin{array}{ll}\text { Absolute energies of ground-state along the } T_{1} \text { mechanisms } & \text { S-17 }\end{array}$

$\begin{array}{ll}\text { Potential energy profiles for 6-4PP formation in the } \mathrm{S}_{0} \text { state } & \mathrm{S}-18\end{array}$

Potential energy profiles for the singlet and triplet states along the $\mathrm{S}_{0}$ reaction S-19

$\begin{array}{ll}\text { Potential energy profiles for 6-4PP formation in the } \mathrm{T}_{1} \text { state } & \mathrm{S}-22\end{array}$

$\begin{array}{lr}\text { Potential energy profiles for two } T_{1} \text { mechanisms } & \text { S-23 }\end{array}$

$\begin{array}{ll}\text { Cartesian coordinates of the optimized geometries } & \text { S-27 }\end{array}$ 
Table S1. Absolute energies in hartrees of the $S_{0}$ stationary points of the 6-4PP formation reaction optimized by B3LYP-D3/cc-pVDZ calculations and multiconfiguration calculations using the cc-pVDZ basis set.

\begin{tabular}{c|c|c|c|c|c}
\cline { 2 - 6 } \multicolumn{5}{c|}{ S $_{\mathbf{0}}$} \\
\hline Structure & E (B3LYP-D3) & E (SA-CASSCF) & E (MS-CASPT2) & E (SA-PDFT) & E (SI-PDFT) \\
\hline SR1 & -2373.533614 & -2363.71469 & -2368.678891 & -2371.857316 & -2371.858098 \\
\hline STS1 & -2373.428866 & -2363.582801 & -2368.582021 & -2371.746135 & -2371.747549 \\
\hline S12 & -2373.497237 & -2363.677362 & -2368.662979 & -2371.841729 & -2371.841625 \\
\hline STS2 & -2373.470151 & -2363.57751 & -2368.624107 & -2371.783998 & -2371.765271 \\
\hline SP1 & -2373.496410 & -2363.666018 & -2368.660808 & -2371.833064 & -2371.834414
\end{tabular}

Table S2. Absolute energies in hartrees of the $T_{1}$ state stationary points optimized by UKS-B3LYP-D3/cc-pVDZ calculations and multiconfiguration calculations using the cc-pVDZ basis set.

\begin{tabular}{|c|c|c|c|c|c|}
\hline & \multicolumn{5}{|c|}{$\mathbf{T}_{1}$} \\
\hline Structure & E (UKS-B3LYP-D3) & E (SA-CASSCF) & E (MS-CASPT2) & E (SA-PDFT) & E (SI-PDFT) \\
\hline R1 & -2373.447238 & -2363.625025 & -2368.58116 & -2371.7625 & -2371.762438 \\
\hline TS1 $1_{\mathrm{CC}}$ & -2373.415479 & -2363.600504 & -2368.5667 & -2371.7373 & -2371.73729 \\
\hline TS1 $_{\text {CS }}$ & -2373.432950 & -2363.594409 & -2368.57194 & -2371.7499 & -2371.749897 \\
\hline $\mathbf{I 1}_{\mathrm{CC}}$ & -2373.419609 & -2363.60217 & -2368.56863 & -2371.7405 & -2371.740343 \\
\hline $\mathbf{I 1}_{\mathrm{CS}}$ & -2373.438895 & -2363.604236 & -2368.58121 & -2371.7588 & -2371.758799 \\
\hline $\mathrm{TS2}_{\mathrm{CC}}$ & -2373.385600 & -2363.547373 & -2368.52558 & -2371.6962 & -2371.696118 \\
\hline TS2 ${ }_{\mathrm{CS}}$ & -2373.385591 & -2363.547377 & -2368.52556 & -2371.6961 & -2371.696108 \\
\hline I2 & -2373.388933 & -2363.570395 & -2368.54778 & -2371.7185 & -2371.718497 \\
\hline TS3 & -2373.393593 & -2363.564048 & -2368.54618 & -2371.7164 & -2371.716366 \\
\hline I3 & -2373.432356 & -2363.611135 & -2368.5839 & -2371.7553 & -2371.755335 \\
\hline TS4 & -2373.422721 & -2363.601306 & -2368.57454 & -2371.7477 & -2371.747726 \\
\hline I4 & -2373.428005 & -2363.600788 & -2368.5799 & -2371.7487 & -2371.748758 \\
\hline TS5 & -2373.395152 & -2363.529284 & -2368.53042 & -2371.7046 & -2371.704635 \\
\hline I5 & -2373.394824 & -2363.545673 & -2368.5311 & -2371.7012 & -2371.701326 \\
\hline P1 & -2373.405600 & -2363.560374 & -2368.539 & -2371.7107 & -2371.711101 \\
\hline
\end{tabular}


Table S3. Absolute energies in hartrees for the ground and vertically excited states as computed by TD-DFT calculations with the CAM-B3LYP exchange-correlation functional and the cc-pVDZ basis set. All energies in this table are reported at the $\mathrm{S}_{0}$ stationary points optimized by B3LYP-D3/cc-pVDZ calculations.

\begin{tabular}{c|c|c|c|c|c}
\hline \multicolumn{5}{c}{ Geometries } \\
\hline State & SR1 & STS1 & SI2 & STS2 & SP1 \\
\hline $\mathbf{S}_{\mathbf{0}}$ & -2373.669753 & -2373.553095 & -2373.639954 & -2373.604307 & -2373.635979 \\
$\mathbf{S}_{\mathbf{1}}$ & -2373.551851 & -2373.514238 & -2373.476411 & -2373.519228 & -2373.485935 \\
$\mathbf{S}_{\mathbf{2}}$ & -2373.514942 & -2373.476557 & -2373.467726 & -2373.504582 & -2373.469812 \\
$\mathbf{S}_{\mathbf{3}}$ & -2373.482696 & -2373.422978 & -2373.448928 & -2373.454696 & -2373.444979 \\
$\mathbf{T}_{\mathbf{1}}$ & -2373.582704 & -2373.564672 & -2373.519391 & -2373.524468 & -2373.537320 \\
$\mathbf{T}_{\mathbf{2}}$ & -2373.563417 & -2373.488281 & -2373.497091 & -2373.519220 & -2373.486916 \\
$\mathbf{T}_{\mathbf{3}}$ & -2373.551800 & -2373.473647 & -2373.483415 & -2373.501848 & -2373.483759
\end{tabular}

Table S4. Absolute energies in hartrees for the ground and vertically excited states as computed by TDA-DFT calculations with the CAM-B3LYP exchange-correlation functional and the cc-pVDZ basis set. All energies in this table are reported at the $\mathrm{S}_{0}$ stationary points optimized by B3LYP-D3/cc-pVDZ calculations.

\begin{tabular}{c|c|c|c|c|c}
\hline \multicolumn{5}{c}{ Geometries } \\
\hline State & SR1 & STS1 & SI2 & STS2 & SP1 \\
\hline $\mathbf{S}_{\mathbf{0}}$ & -2373.6698 & -2373.5531 & -2373.64 & -2373.6043 & -2373.636 \\
$\mathbf{S}_{\mathbf{1}}$ & -2373.5512 & -2373.5076 & -2373.4755 & -2373.5187 & -2373.4774 \\
$\mathbf{S}_{\mathbf{2}}$ & -2373.5049 & -2373.4752 & -2373.4662 & -2373.5023 & -2373.4681 \\
$\mathbf{S}_{\mathbf{3}}$ & -2373.4821 & -2373.417 & -2373.4479 & -2373.4472 & -2373.4448 \\
$\mathbf{T}_{\mathbf{1}}$ & -2373.5736 & -2373.5382 & -2373.5085 & -2373.5233 & -2373.531 \\
$\mathbf{T}_{\mathbf{2}}$ & -2373.562 & -2373.4867 & -2373.4951 & -2373.5179 & -2373.4844 \\
$\mathbf{T}_{\mathbf{3}}$ & -2373.5417 & -2373.4651 & -2373.4803 & -2373.4971 & -2373.4753 \\
\end{tabular}


Table S5. Absolute energies in hartrees for the ground and vertically excited states as computed by MS-CASPT2(12,11)/cc-pVDZ. All energies in this table are reported at the $\mathrm{S}_{0}$ stationary points optimized by B3LYP-D3/cc-pVDZ calculations.

\begin{tabular}{c|c|c|c|c|c}
\multicolumn{1}{c}{} \\
\cline { 2 - 6 } State & SR1 & STS1 & SI2 & STS2 & SP1 \\
\hline $\mathbf{S}_{\mathbf{0}}$ & -2368.6789 & -2368.582 & -2368.663 & -2368.6241 & -2368.6608 \\
$\mathbf{S}_{\mathbf{1}}$ & -2368.5676 & -2368.5417 & -2368.4898 & -2368.5469 & -2368.5055 \\
$\mathbf{S}_{\mathbf{2}}$ & -2368.5179 & -2368.5076 & -2368.4675 & -2368.5344 & -2368.4898 \\
$\mathbf{S}_{\mathbf{3}}$ & -2368.4613 & -2368.4772 & -2368.441 & -2368.4391 & -2368.4102 \\
$\mathbf{T}_{\mathbf{1}}$ & -2368.5697 & -2368.5514 & -2368.5109 & -2368.5441 & -2368.5255 \\
$\mathbf{T}_{\mathbf{2}}$ & -2368.5685 & -2368.5099 & -2368.5026 & -2368.5346 & -2368.4934 \\
$\mathbf{T}_{\mathbf{3}}$ & -2368.5286 & -2368.4723 & -2368.4579 & -2368.4338 & -2368.4514
\end{tabular}

Table S6. Absolute energies in hartrees for the ground and vertically excited states as computed by CAS(12,11)-tPBE/cc-pVDZ All energies in this table are reported at the $\mathrm{S}_{0}$ stationary points optimized by B3LYP-D3/cc-pVDZ calculations.

\begin{tabular}{c|c|c|c|c|c}
\multicolumn{5}{c}{} & \multicolumn{5}{c}{ Geometries } \\
\hline State & SR1 & STS1 & SI2 & STS2 & SP1 \\
\hline $\mathbf{S}_{\mathbf{0}}$ & -2371.8573 & -2371.7461 & -2371.8417 & -2371.784 & -2371.8331 \\
$\mathbf{S}_{\mathbf{1}}$ & -2371.7463 & -2371.7188 & -2371.6755 & -2371.7121 & -2371.6584 \\
$\mathbf{S}_{\mathbf{2}}$ & -2371.7173 & -2371.6832 & -2371.6553 & -2371.7312 & -2371.6768 \\
$\mathbf{S}_{\mathbf{3}}$ & -2371.6343 & -2371.6493 & -2371.6325 & -2371.599 & -2371.5894 \\
$\mathbf{T}_{\mathbf{1}}$ & -2371.752 & -2371.7226 & -2371.6884 & -2371.7135 & -2371.6977 \\
$\mathbf{T}_{\mathbf{2}}$ & -2371.7502 & -2371.6805 & -2371.6823 & -2371.7044 & -2371.665 \\
$\mathbf{T}_{\mathbf{3}}$ & -2371.6932 & -2371.6433 & -2371.6562 & -2371.5986 & -2371.62
\end{tabular}


Table S7. Absolute energies in hartrees for the ground and vertically excited states as computed by SI-CAS(12,11)-tPBE/cc-pVDZ. All energies in this table are reported at the S0 stationary points optimized by B3LYP-D3/cc-pVDZ calculations.

\begin{tabular}{c|c|c|c|c|c}
\multicolumn{2}{c}{ Geometries } \\
\hline State & SR1 & STS1 & SI2 & STS2 & SP1 \\
\hline $\mathbf{S}_{\mathbf{0}}$ & -2371.8581 & -2371.7475 & -2371.8416 & -2371.7653 & -2371.8344 \\
$\mathbf{S}_{\mathbf{1}}$ & -2371.7463 & -2371.7182 & -2371.6755 & -2371.712 & -2371.6582 \\
$\mathbf{S}_{\mathbf{2}}$ & -2371.7161 & -2371.682 & -2371.6552 & -2371.702 & -2371.6772 \\
$\mathbf{S}_{\mathbf{3}}$ & -2371.6343 & -2371.6495 & -2371.6325 & -2371.5988 & -2371.5886 \\
$\mathbf{T}_{\mathbf{1}}$ & -2371.7531 & -2371.7268 & -2371.686 & -2371.714 & -2371.7067 \\
$\mathbf{T}_{\mathbf{2}}$ & -2371.7491 & -2371.6814 & -2371.6875 & -2371.7058 & -2371.667 \\
$\mathbf{T}_{\mathbf{3}}$ & -2371.6927 & -2371.641 & -2371.6542 & -2371.6018 & -2371.6212 \\
\end{tabular}


Table S8. Multiconfiguration calculations using cc-pVDZ of the absolute energies in hartrees for the ground-state of the $T_{1}$ state stationary points optimized by UKS-B3LYPD3/cc-pVDZ calculations.

\begin{tabular}{|c|c|c|}
\cline { 2 - 3 } & \multicolumn{2}{|c}{ S $_{\mathbf{0}}$} \\
\hline Structure & E (UKS-B3LYP-D3) & E (MS-CASPT2) \\
\hline R1 & -2373.51846 & -2368.660311 \\
\hline TS1CC & -2373.41308 & -2368.57601 \\
\hline TS1CS & -2373.46446 & -2368.617513 \\
\hline I1CC & -2373.40566 & -2368.571387 \\
\hline I1CS & -2373.44574 & -2368.602572 \\
\hline TS2 $C C$ & -2373.46418 & -2368.631452 \\
\hline TS2 $C S$ & -2373.46416 & -2368.631423 \\
\hline I2 & -2373.51846 & -2368.581308 \\
\hline TS3 & -2373.45882 & -2368.626429 \\
\hline I3 & -2373.46759 & -2368.623025 \\
\hline TS4 & -2373.45344 & -2368.606485 \\
\hline I4 & -2373.46420 & -2368.618008 \\
\hline TS5 & -2373.47722 & -2368.635524 \\
\hline I5 & -2373.47525 & -2368.640048 \\
\hline P1 & -2373.48348 & -2368.649255 \\
\hline
\end{tabular}



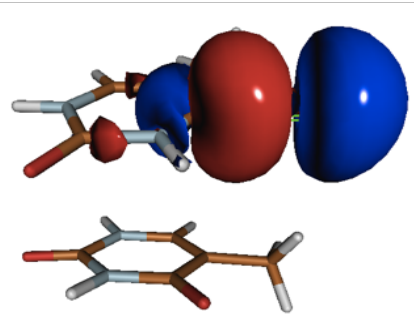

$\sigma_{\mathrm{CS}}$

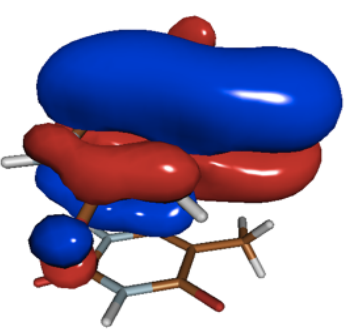

$\pi_{\mathrm{CS}} \quad 1.83$

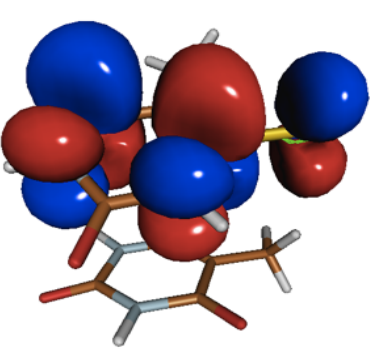

$\pi^{*}{ }_{\mathrm{CC}} \quad 0.72$
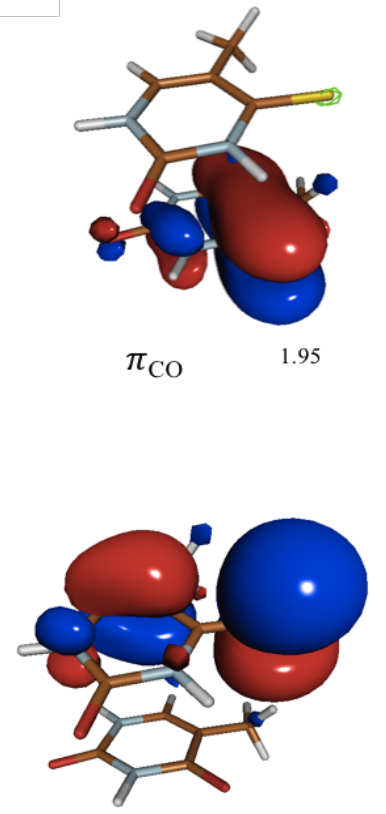

$\pi_{\mathrm{CC}}$

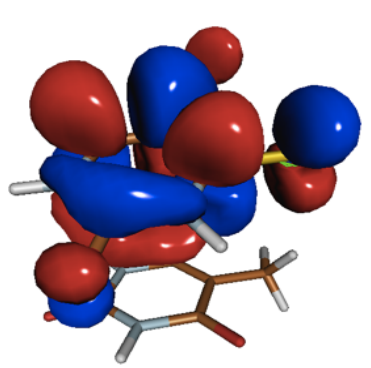

$\pi^{*}{ }_{\mathrm{CS}} \quad 0.24$
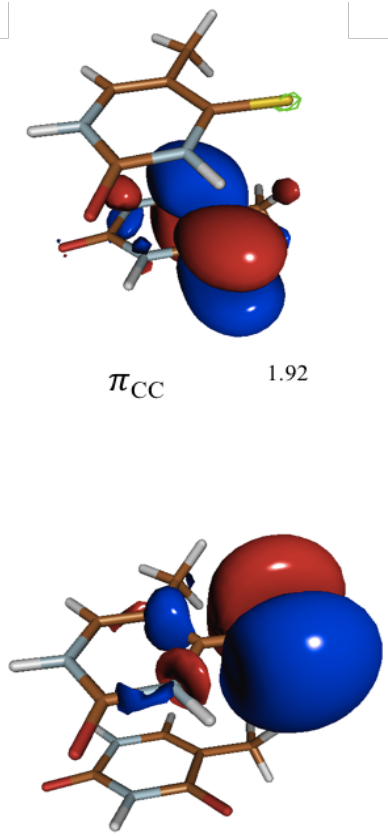

$n_{\mathrm{CS}} \quad 1.50$

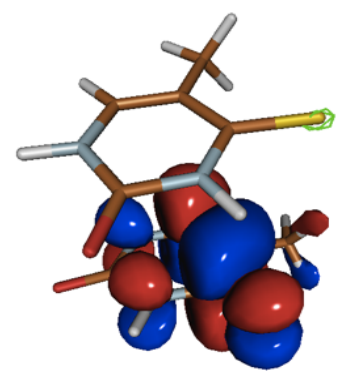

$\pi^{*}{ }_{\mathrm{CC}}$

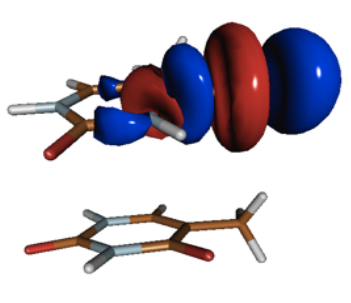

$\sigma^{*}{ }_{\mathrm{CS}} \quad 0.03$

Figure S1. SA-CASSCF/cc-pVDZ orbitals with their occupation numbers included in the $(12,11)$ active space employed in the multireference calculations at the ground-state SR1 structure. The sugar-phosphate DNA backbone has been omitted from the figure for clarity. 
Table S9. M values for the multireference M diagnostic obtained for the lowest-lying singlet and triplet electronic states along the $\mathrm{S}_{0} 6-4 \mathrm{PP}$ formation mechanism. Values calculated using natural occupancies of SA-CASSCF/cc-pVDZ wave function.

\begin{tabular}{|c|c|c|c|c|c|c|c|}
\hline \multicolumn{7}{|c|}{ M value } \\
\hline Structure & $\mathrm{S}_{0}$ & $\mathrm{~S}_{1}$ & $\mathrm{~S}_{2}$ & $\mathrm{~S}_{3}$ & $\mathrm{~T}_{1}$ & $\mathrm{~T}_{2}$ & $\mathrm{~T}_{3}$ \\
\hline SR1 & 0.048 & 0.547 & 0.554 & 0.744 & 0.552 & 0.556 & 0.597 \\
\hline STS1 & 0.234 & 0.593 & 0.629 & 0.577 & 0.557 & 0.630 & 0.873 \\
\hline SI2 & 0.015 & 0.579 & 0.617 & 0.666 & 0.518 & 0.589 & 0.594 \\
\hline STS2 & 0.036 & 0.543 & 0.544 & 0.773 & 0.545 & 0.546 & 0.756 \\
\hline SP1 & 0.081 & 0.589 & 0.542 & 0.798 & 0.554 & 0.594 & 0.884 \\
\hline
\end{tabular}

Table S10. M values for the multireference M diagnostic obtained for the lowest-lying triplet electronic state along the $\mathrm{T}_{1} 6$-4PP formation mechanism. Values calculated using natural occupancies of SA-CASSCF/cc-pVDZ wave function.

\begin{tabular}{|c|c|}
\hline & M value \\
\hline Structure & $\mathrm{T}_{1}$ \\
\hline R1 & 0.588 \\
\hline TS1cc & 0.576 \\
\hline TS1cs & 0.611 \\
\hline I1cC & 0.578 \\
\hline I1 cs & 0.548 \\
\hline TS2 & 0.579 \\
\hline TS2 Cs & 0.579 \\
\hline I2 & 0.519 \\
\hline TS3 & 0.523 \\
\hline I3 & 0.530 \\
\hline TS4 & 0.547 \\
\hline I4 & 0.548 \\
\hline TS5 & 0.546 \\
\hline I5 & 0.548 \\
\hline P1 & 0.551 \\
\hline
\end{tabular}


Table S11. Absolute energies in hartrees for the ground and vertically excited states as computed by SA-CASSCF $(12,11) / c c-p V D Z$. All energies in this table are reported at the $\mathrm{S}_{0}$ stationary points optimized by B3LYP-D3/cc-pVDZ calculations.

\begin{tabular}{c|c|c|c|c|c}
\multicolumn{2}{c}{ Geometries } \\
\hline State & SR1 & STS1 & SI2 & STS2 & SP1 \\
\hline $\mathbf{S}_{\mathbf{0}}$ & -2363.7147 & -2363.5828 & -2363.6774 & -2363.5775 & -2363.666 \\
$\mathbf{S}_{\mathbf{1}}$ & -2363.6074 & -2363.5491 & -2363.4883 & -2363.5467 & -2363.4945 \\
$\mathbf{S}_{\mathbf{2}}$ & -2363.5302 & -2363.5185 & -2363.4208 & -2363.5251 & -2363.4495 \\
$\mathbf{S}_{\mathbf{3}}$ & -2363.492 & -2363.463 & -2363.4139 & -2363.4318 & -2363.4174 \\
$\mathbf{T}_{\mathbf{1}}$ & -2363.6096 & -2363.5615 & -2363.5278 & -2363.5552 & -2363.5481 \\
$\mathbf{T}_{\mathbf{2}}$ & -2363.6083 & -2363.5275 & -2363.5059 & -2363.5456 & -2363.5133 \\
$\mathbf{T}_{\mathbf{3}}$ & -2363.5694 & -2363.4795 & -2363.4518 & -2363.4388 & -2363.4771
\end{tabular}




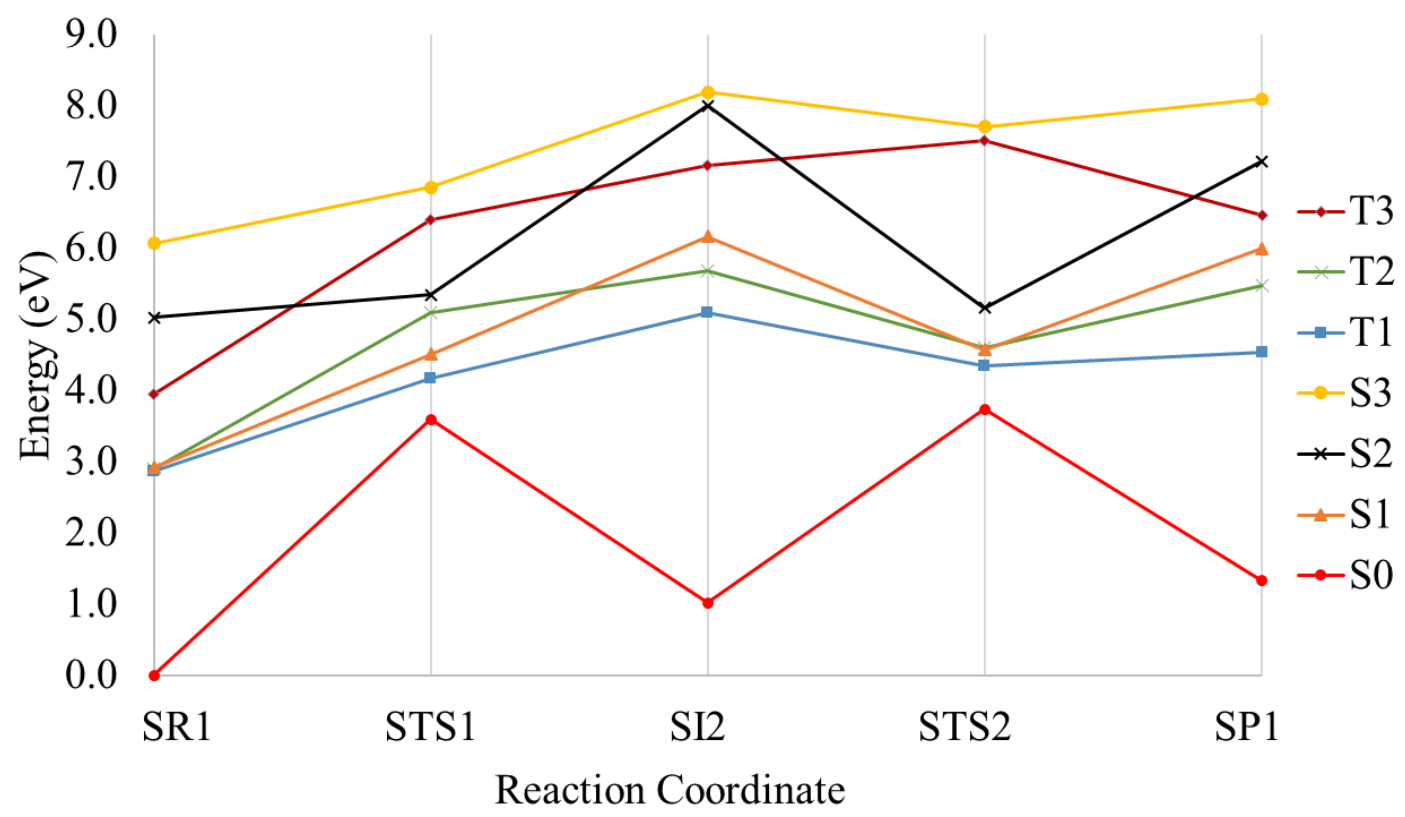

Figure S2. Energy profiles for the lowest singlet and triplet states of the 6-4PP formation mechanism computed at the ground-state stationary points by SA-CASSCF $(12,11) / \mathrm{cc}-$ pVDZ. Energies are in eV relative to the SR1 ground-state equilibrium geometry.

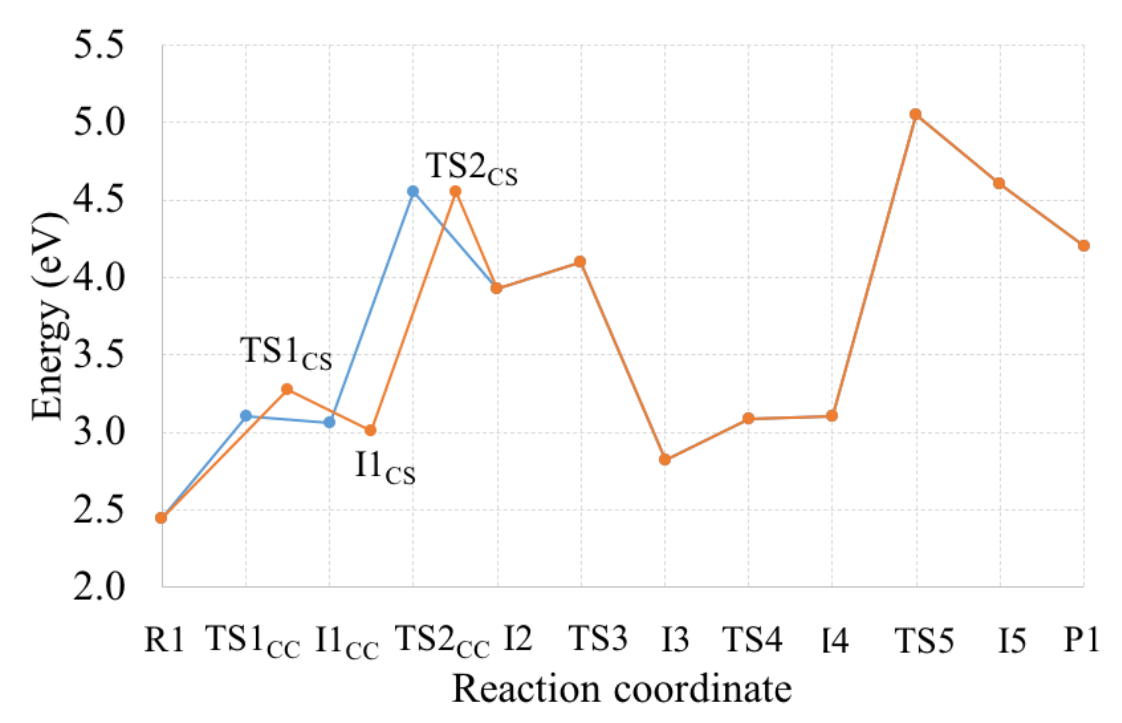

Figure S3. $\mathrm{T}_{1}$ Potential energy profiles for the CC-CS (blue line) and the CS-CC triplet mechanism (orange line) CASSCF(12,11)/cc-pVDZ. 
In order to analyze the effect of polarization functions, the potential energy profile of $S_{0}$ at MS-CASPT2 level has been calculated using both the cc-pVDZ basis set (the same basis set employed in the DFT and TD-DFT calculations) and the ANO-RCC-DVZP basis set, which incorporates polarization functions to the ANO-RCC-DVZ basis set previously used in our multiconfigurational calculations.

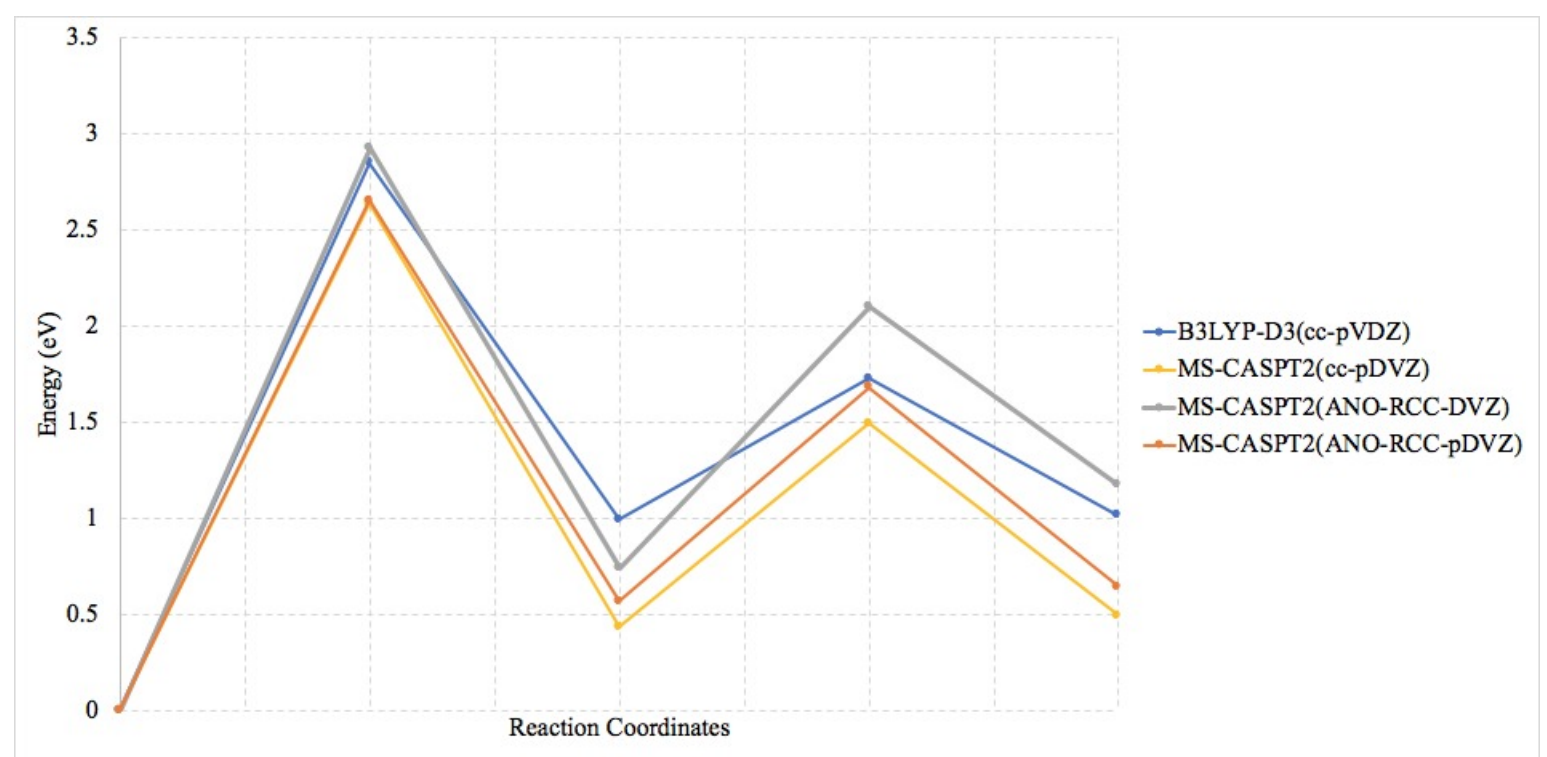

Figure S4. Potential energy profiles for 6-4PP formation in the $\mathrm{S}_{0}$ state at different levels of theory: B3LYP-D3/cc-pVDZ: blue line; MS-CASPT2/cc-pVDZ: yellow line; MSCASPT2/ANO-RCC-VDZ: grey line; MS-CASPT2/ANO-RCC-VDZP: orange line. Energies are in $\mathrm{eV}$ relative to the SR1 ground-state equilibrium structure. All geometries in this profile were optimized at the B3LYP-D3 level.

The results in fig. S4 reveal that all the potential energy curves present the same qualitative shape; that is, the first step of the reaction mechanism, showing approach between the two nucleobases, presents an energy barrier lying about $0.8-1 \mathrm{eV}$ higher in energy than the second step, which is responsible for the concerted transfer of the S and $\mathrm{H}$ atoms. All the computational protocols predict the intermediate and final products as less stable than the initial product with similar energies except for the CASPT2/ANORCC-VDZ, which predicts the final product to be higher in energy by $0.4 \mathrm{eV}$. 
Also, almost negligible differences are found between the CASPT2 results computed with the two basis sets including polarization functions, ANO-RCC-VDZP and cc-pVDZ. The main differences are concentrated in the last step of the reaction where the Dunning basis stabilizes the energy of the minima by about $0.15 \mathrm{eV}$ and also stabilizes the energy of the TS state connecting them by about $0.19 \mathrm{eV}$.

The polarization function has an overall stabilizing effect along the complete potential energy curve, this effect is more pronounced for the second step of the reaction.

The comparison of the B3LYP and CASPT2 results, here calculated with the same basis set, cc-pVDZ, strengthens the conclusion that DFT overestimates the energy of the SI2 intermediate. We also find that the single-configuration approach also overestimates the energies of the two transition states, in other words, the B3LYP potential energy curve lies above the MS-CASPT2 profile. 


\section{Results with the ANO-RCC-VDZ Basis Set}

Table S12. Single point energies (hartrees) of the $S_{0}$ stationary points of the 6-4PP formation reaction with all methods using ANO-RCC-VDZ basis set.

\begin{tabular}{c|c|c|c|c|c}
\cline { 2 - 6 } \multicolumn{5}{c|}{ S } & \\
\hline Structure & E (B3LYP-D3) & E (SA-CASSCF) & E (MS-CASPT2) & E (MC-PDFT) & E (SI-PDFT) \\
\hline SR1 & -2373.533614 & -2365.911315 & -2369.158354 & -2374.537802 & -2374.538873 \\
STS1 & -2373.428866 & -2365.784795 & -2369.050742 & -2374.426430 & -2374.428354 \\
S12 & -2373.497237 & -2365.871097 & -2369.131146 & -2374.522035 & -2374.521957 \\
\hline STS2 & -2373.470151 & -2365.765810 & -2369.081343 & -2374.462223 & -2374.443114 \\
SP1 & -2373.496410 & -2365.840101 & -2369.115211 & -2374.503675 & -2374.504593
\end{tabular}

Table S13. Single point energies (hartrees) of the $\mathrm{T}_{1}$ stationary points of the 6-4PP formation reaction with all methods using ANO-RCC-VDZ basis set.

\begin{tabular}{|c|c|c|c|c|c|}
\hline & \multicolumn{5}{|c|}{$\mathbf{T}_{1}$} \\
\hline Structure & E (UKS-B3LYP-D3) & E (SA-CASSCF) & E (MS-CASPT2) & E (SA-PDFT) & E (SI-PDFT) \\
\hline R1 & -2373.447238 & -2365.900555 & -2369.068393 & -2374.444348 & -2374.444332 \\
\hline TS1 $_{\text {CC }}$ & -2373.415479 & -2365.811628 & -2369.051163 & -2374.426779 & -2374.426820 \\
\hline TS1 $_{\text {CS }}$ & -2373.432950 & -2365.833892 & -2369.046392 & -2374.444348 & -2374.428443 \\
\hline $\mathbf{I 1}_{\mathbf{C C}}$ & -2373.419609 & -2365.809673 & -2369.054994 & -2374.43162 & -2374.431459 \\
\hline I1 $1_{\mathrm{CS}}$ & -2373.438895 & -2365.808617 & -2369.048317 & -2374.428453 & -2374.428701 \\
\hline TS2 & -2373.385600 & -2365.837990 & -2368.993035 & -2374.373240 & -2374.373219 \\
\hline TS2 $_{\text {CS }}$ & -2373.385591 & -2365.838010 & -2368.993057 & -2374.428738 & -2374.373223 \\
\hline I2 & -2373.388933 & -2365.769500 & -2369.010028 & -2374.391250 & -2374.391251 \\
\hline TS3 & -2373.393593 & -2365.825804 & -2369.006091 & -2374.383745 & -2374.383745 \\
\hline I3 & -2373.432356 & -2365.821212 & -2369.063671 & -2374.443616 & -2374.443615 \\
\hline TS4 & -2373.422721 & -2365.798336 & -2369.052908 & -2374.434947 & -2374.434948 \\
\hline I4 & -2373.428005 & -2365.805816 & -2369.056194 & -2374.435195 & -2374.435204 \\
\hline TS5 & -2373.395152 & -2365.775417 & -2368.990601 & -2374.389175 & -2374.395338 \\
\hline I5 & -2373.394824 & -2365.804841 & -2368.985951 & -2374.370098 & -2374.370152 \\
\hline P1 & -2373.405600 & -2365.823038 & -2368.992022 & -2374.377445 & -2374.377660 \\
\hline
\end{tabular}


Table S14. Absolute energies in hartrees for the ground and vertically excited states as computed by MS-CASPT2(12,11)/ANO-RCC-VDZ. All energies in this table are reported at the $\mathrm{S}_{0}$ stationary points optimized by B3LYP-D3/cc-pVDZ calculations.

\begin{tabular}{c|c|c|l|l|l}
\cline { 2 - 6 } \multicolumn{5}{c}{ Geometries } \\
\hline State & SR1 & STS1 & SI2 & STS2 & SP1 \\
\hline $\mathbf{S}_{\mathbf{0}}$ & -2369.149792 & -2369.050742 & -2369.131146 & -2369.081343 & -2369.115211 \\
$\mathbf{S}_{\mathbf{1}}$ & -2369.043682 & -2369.018649 & -2368.954008 & -2369.010918 & -2368.954981 \\
$\mathbf{S}_{\mathbf{2}}$ & -2368.995191 & -2368.982911 & -2368.925628 & -2369.000848 & -2368.944687 \\
$\mathbf{S}_{\mathbf{3}}$ & -2368.929817 & -2368.945554 & -2368.907918 & -2368.899994 & -2368.857121 \\
$\mathbf{T}_{\mathbf{1}}$ & -2369.044322 & -2369.023359 & -2368.971723 & -2369.007402 & -2368.975032 \\
$\mathbf{T}_{\mathbf{2}}$ & -2369.043231 & -2368.984974 & -2368.965755 & -2368.999537 & -2368.949101 \\
$\mathbf{T}_{\mathbf{3}}$ & -2368.994790 & -2368.942598 & -2368.908595 & -2368.893291 & -2368.900647
\end{tabular}

Table S15. Absolute energies in hartrees for the ground and vertically excited states as computed by CAS(12,11)-tPBE/ANO-RCC-VDZ. All energies in this table are reported at the $\mathrm{S}_{0}$ stationary points optimized by B3LYP-D3/cc-pVDZ calculations.

\begin{tabular}{c|c|c|c|c|c}
\hline \multicolumn{5}{c}{ Geometries } \\
\hline State & SR1 & STS1 & SI2 & STS2 & SP1 \\
\hline $\mathbf{S}_{\mathbf{0}}$ & -2374.537802 & -2374.426430 & -2374.522035 & -2374.462223 & -2374.503675 \\
$\mathbf{S}_{\mathbf{1}}$ & -2374.426707 & -2374.404339 & -2374.353181 & -2374.389839 & -2374.330679 \\
$\mathbf{S}_{\mathbf{2}}$ & -2374.397651 & -2374.370941 & -2374.311775 & -2374.416546 & -2374.339395 \\
$\mathbf{S}_{\mathbf{3}}$ & -2374.31042 & -2374.330787 & -2374.331101 & -2374.273535 & -2374.256124 \\
$\mathbf{T}_{\mathbf{1}}$ & -2374.427432 & -2374.404538 & -2374.357488 & -2374.389287 & -2374.362512 \\
$\mathbf{T}_{\mathbf{2}}$ & -2374.428353 & -2374.365152 & -2374.355370 & -2374.381755 & -2374.33769 \\
$\mathbf{T}_{\mathbf{3}}$ & -2374.382912 & -2374.322811 & -2374.293245 & -2374.271275 & -2374.284188
\end{tabular}


Table S16. Absolute energies in hartrees for the ground and vertically excited states as computed by SI-CAS(12,11)-tPBE/ANO-RCC-VDZ. All energies in this table are reported at the $\mathrm{S}_{0}$ stationary points optimized by B3LYP-D3/cc-pVDZ calculations.

\begin{tabular}{c|c|c|c|c|c}
\cline { 2 - 6 } \multicolumn{5}{c}{ Geometries } \\
\hline State & SR1 & STS1 & SI2 & STS2 & SP1 \\
\hline $\mathbf{S}_{\mathbf{0}}$ & -2374.538873 & -2374.428354 & -2374.521957 & -2374.443114 & -2374.504593 \\
$\mathbf{S}_{\mathbf{1}}$ & -2374.426726 & -2374.403493 & -2374.353182 & -2374.389774 & -2374.339719 \\
$\mathbf{S}_{\mathbf{2}}$ & -2374.396656 & -2374.369782 & -2374.331017 & -2374.382353 & -2374.330677 \\
$\mathbf{S}_{\mathbf{3}}$ & -2374.310437 & -2374.330955 & -2374.311784 & -2374.273334 & -2374.255804 \\
$\mathbf{T}_{\mathbf{1}}$ & -2374.428431 & -2374.404438 & -2374.357567 & -2374.389300 & -2374.36269 \\
$\mathbf{T}_{\mathbf{2}}$ & -2374.427249 & -2374.365234 & -2374.355315 & -2374.381741 & -2374.337765 \\
$\mathbf{T}_{\mathbf{3}}$ & -2374.382913 & -2374.322843 & -2374.293245 & -2374.271277 & -2374.283964 \\
\hline
\end{tabular}

Table S17. Absolute energies in hartrees for the ground and vertically excited states as computed by SA-CASSCF(12,11)/ANO-RCC-VDZ. All energies in this table are reported at the $\mathrm{S}_{0}$ stationary points optimized by B3LYP-D3/cc-pVDZ calculations.

\begin{tabular}{c|c|c|c|c|c}
\hline \multicolumn{5}{c}{} & \multicolumn{5}{c}{ Geometries } \\
\hline State & SR1 & STS1 & SI2 & STS2 & SP1 \\
\hline $\mathbf{S}_{\mathbf{0}}$ & -2365.911315 & -2365.784795 & -2365.871097 & -2365.765816 & -2365.840101 \\
$\mathbf{S}_{\mathbf{1}}$ & -2365.808883 & -2365.751249 & -2365.682209 & -2365.741209 & -2365.677275 \\
$\mathbf{S}_{\mathbf{2}}$ & -2365.729346 & -2365.723362 & -2365.619391 & -2365.719574 & -2365.618697 \\
$\mathbf{S}_{\mathbf{3}}$ & -2365.689793 & -2365.664778 & -2365.611293 & -2365.624149 & -2365.588442 \\
$\mathbf{T}_{\mathbf{1}}$ & -2365.810377 & -2365.763433 & -2365.720467 & -2365.749093 & -2365.721510 \\
$\mathbf{T}_{\mathbf{2}}$ & -2365.808361 & -2365.732282 & -2365.696934 & -2365.741661 & -2365.696552 \\
$\mathbf{T}_{\mathbf{3}}$ & -2365.765533 & -2365.681439 & -2365.667212 & -2365.630965 & -2365.649906
\end{tabular}


Table $\mathrm{S} 18$. Absolute energies in hartrees for the $\mathrm{T}_{1}$ state stationary points with UKSB3LYP-D3/ANO-RCC-VDZ and CASPT2/ANO-RCC-VDZ.

\begin{tabular}{|c|c|c|}
\hline & \multicolumn{2}{|c|}{$\mathbf{S}_{0}$} \\
\hline Structure & E (UKS-B3LYP-D3) & E (MS-CASPT2) \\
\hline R1 & -2373.51846 & -2369.14721 \\
\hline TS1 & -2373.41308 & -2369.05816 \\
\hline TS1cs & -2373.46446 & -2369.09388 \\
\hline $\mathbf{I} 1_{\mathrm{CC}}$ & -2373.40566 & -2369.05626 \\
\hline $\mathbf{I 1}_{\mathrm{CS}}$ & -2373.44574 & -2369.07061 \\
\hline $\mathbf{T S} 2_{\mathrm{CC}}$ & -2373.46418 & -2369.10349 \\
\hline $\mathbf{T S} 2_{\mathrm{CS}}$ & -2373.46416 & -2369.10350 \\
\hline I2 & -2373.51846 & -2369.04405 \\
\hline TS3 & -2373.45882 & -2369.09323 \\
\hline I3 & -2373.46759 & -2369.09470 \\
\hline TS4 & -2373.45344 & -2369.07437 \\
\hline I4 & -2373.46420 & -2369.08780 \\
\hline TS5 & -2373.47722 & -2369.09022 \\
\hline I5 & -2373.47525 & -2369.09657 \\
\hline P1 & -2373.48348 & -2369.10562 \\
\hline
\end{tabular}




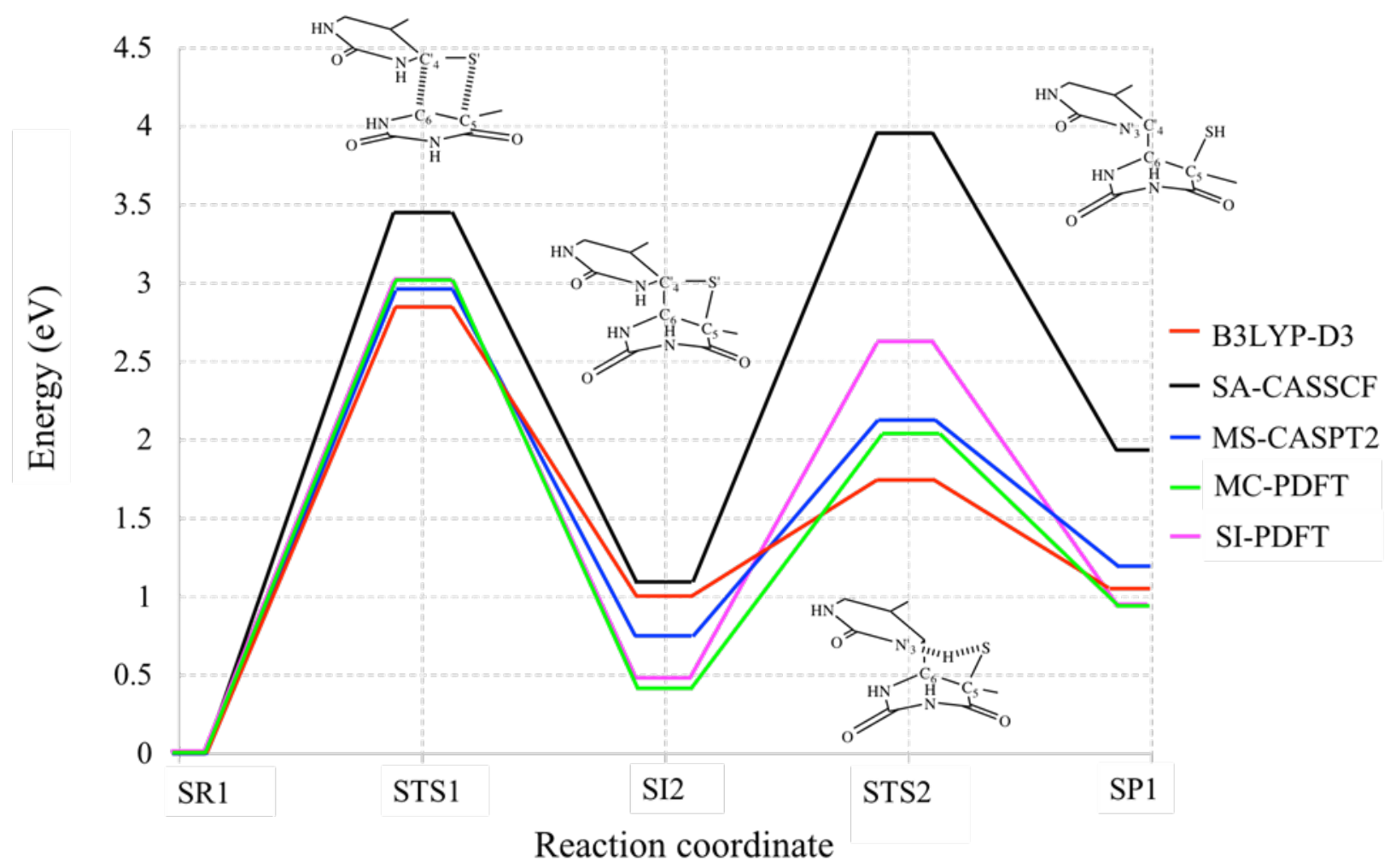

Figure S5: Potential energy profiles for 6-4PP formation in the $\mathrm{S}_{0}$ state at different levels of theory: B3LYP-D3/cc-pVDZ: red line; SA-CASSCF/ANO-RCC-VDZ: black line; MS-CASPT2/ANO-RCC-VDZ: blue line; MC-PDFT/ANO-RCC-VDZ: green line and SI-PDFT/ANO-RCC-VDZ: pink line. Energies are in eV relative to the SR1 ground-state equilibrium structure. All geometries in this profile were optimized at the B3LYP-D3 level. 


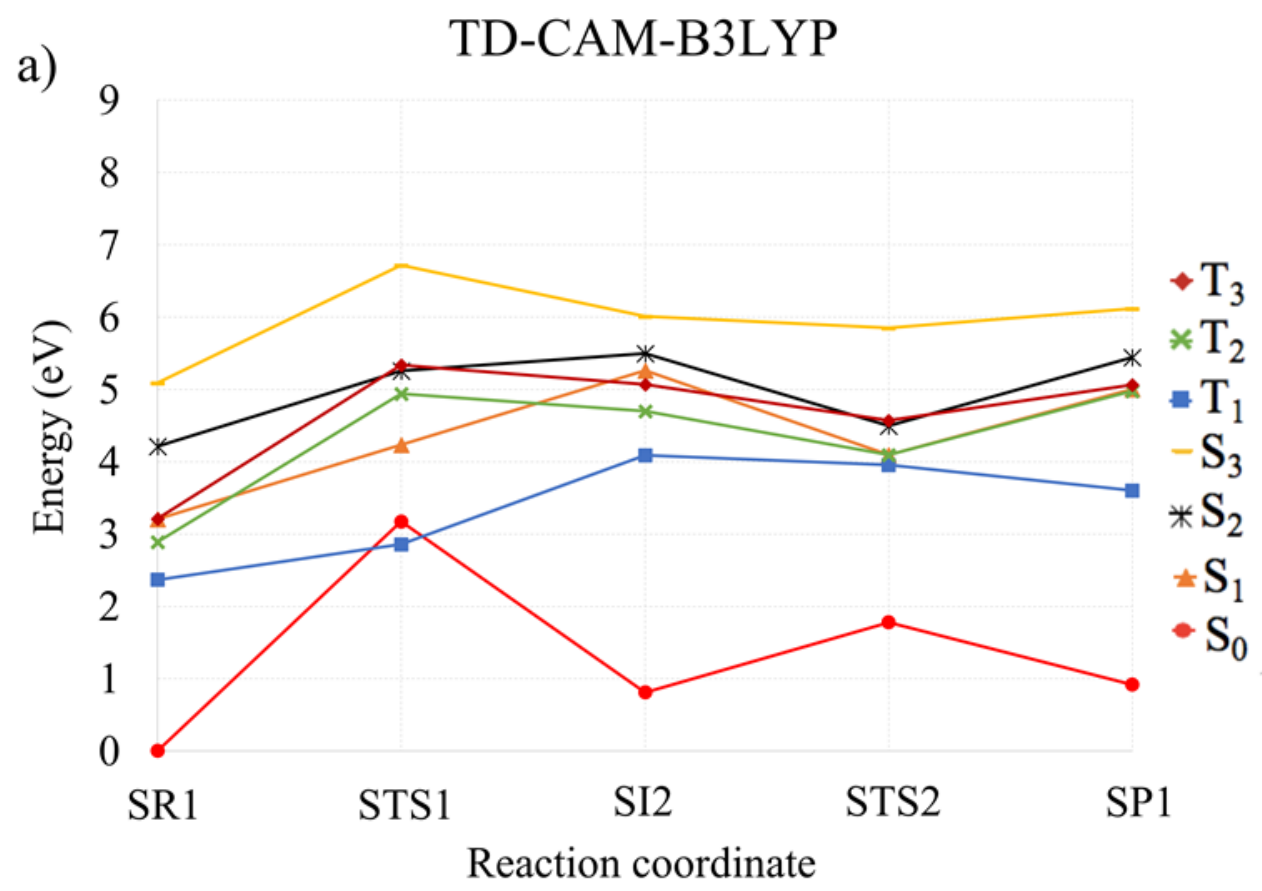

b)

\section{MS-CASPT2}

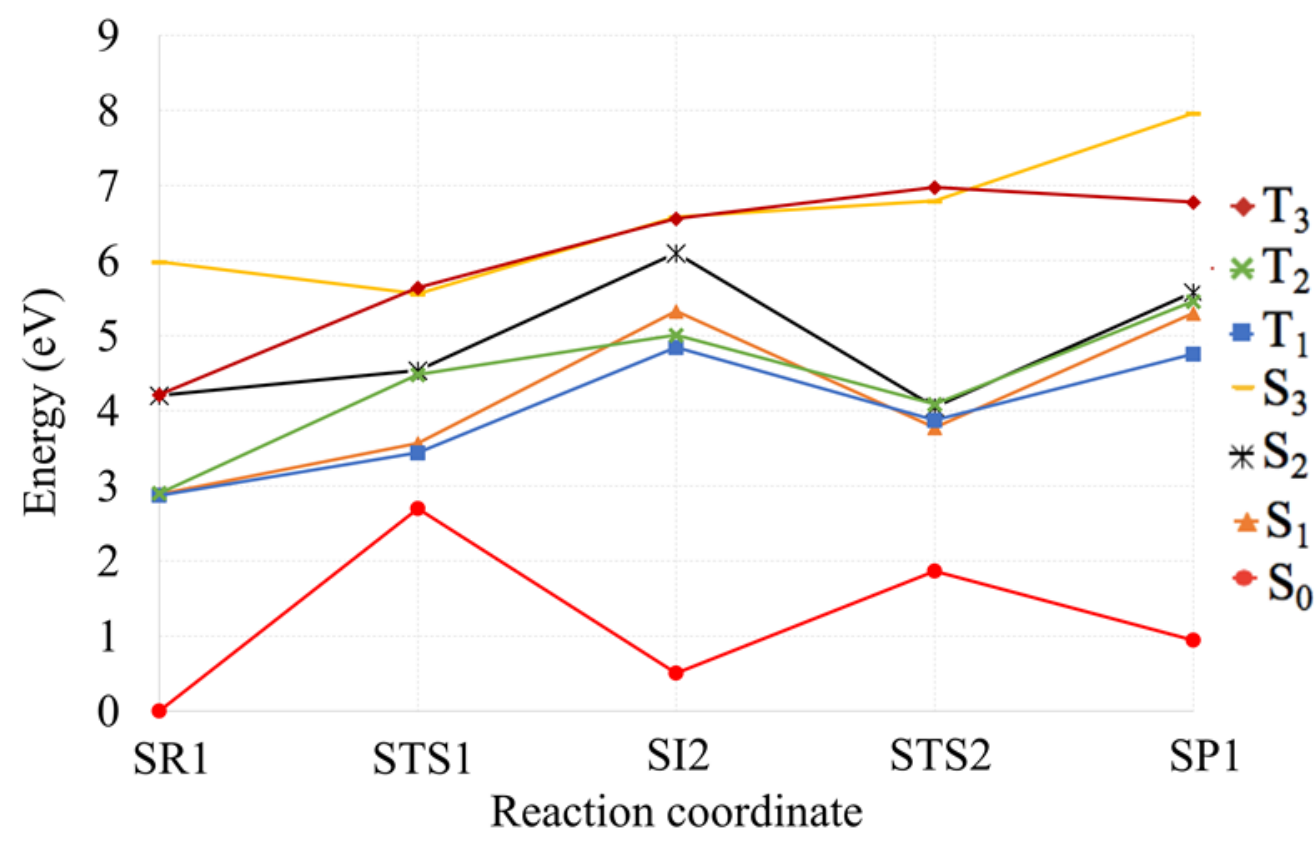



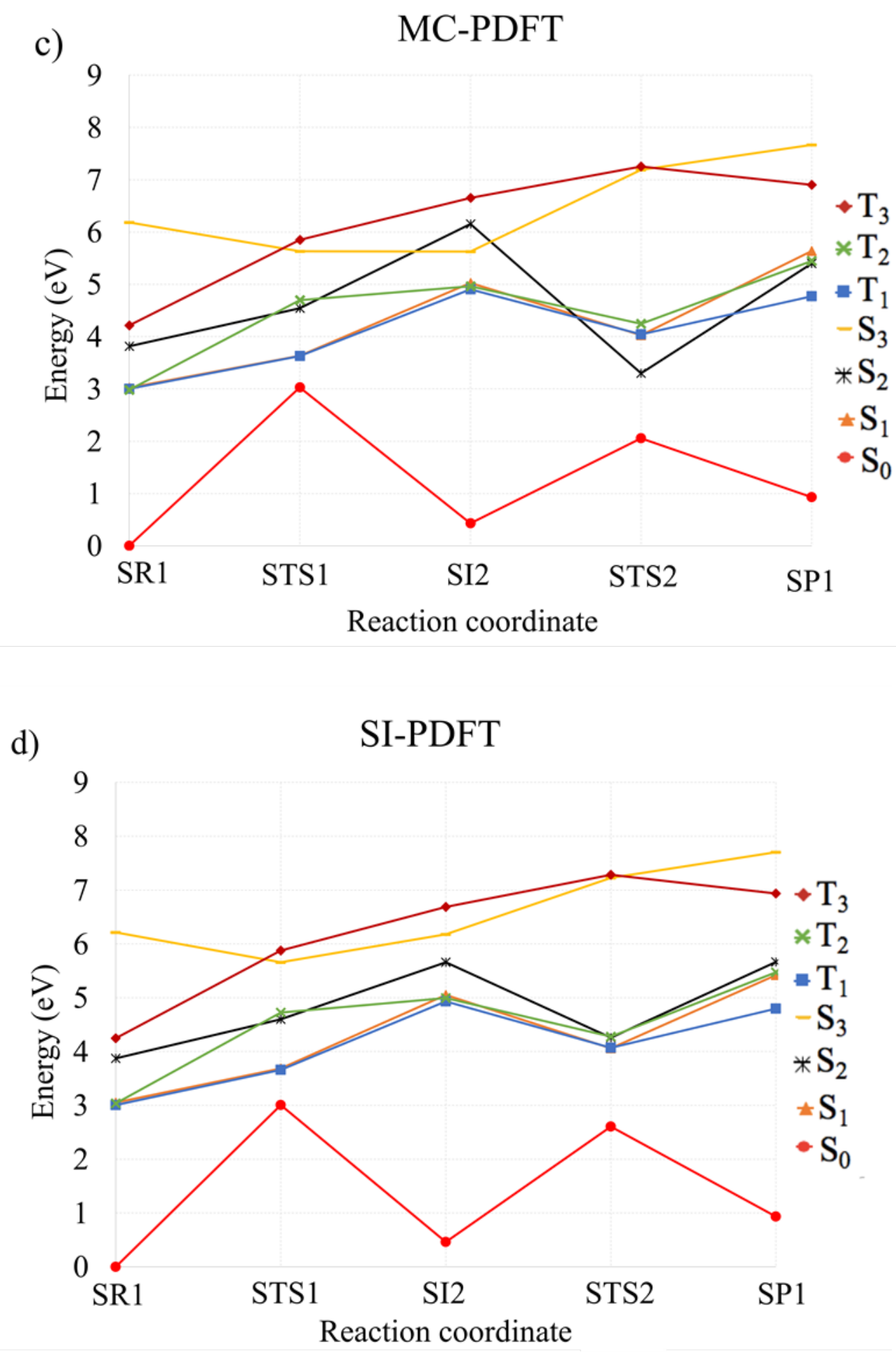

Figure S6: Potential energy profiles for the lowest singlet and triplet states along the ground-state reaction coordinate leading to 6-4PP formation, computed at various levels of theory. (a) TD-CAM-B3LYP/cc-pVDZ, (b) MS-CASPT2/ANO-RCC-VDZ, (c) MCPDFT/ANO-RCC-VDZ, (d) SI-PDFT/ANO-RCC-VDZ. Energies are in eV relative to 
the SR1 ground-state equilibrium geometry. All geometries in this profile were optimized at the B3LYP-D3 level (same as in Fig. S5).

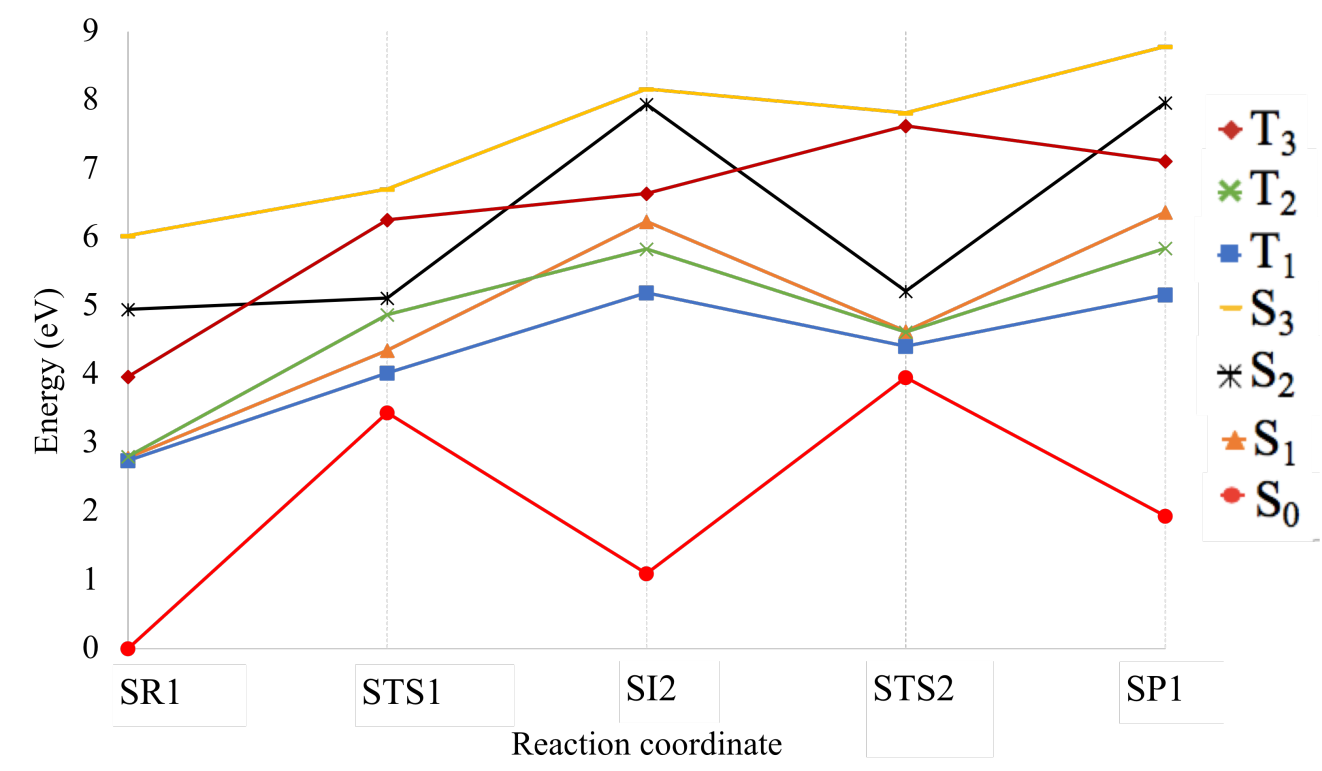

Figure S7. Energy profiles for the lowest singlet and triplet states of the 6-4PP formation mechanism computed at the ground-state stationary points by SA-CASSCF/ANO-RCCVDZ. Energies are in $\mathrm{eV}$ relative to the SR1 ground-state equilibrium geometry. 

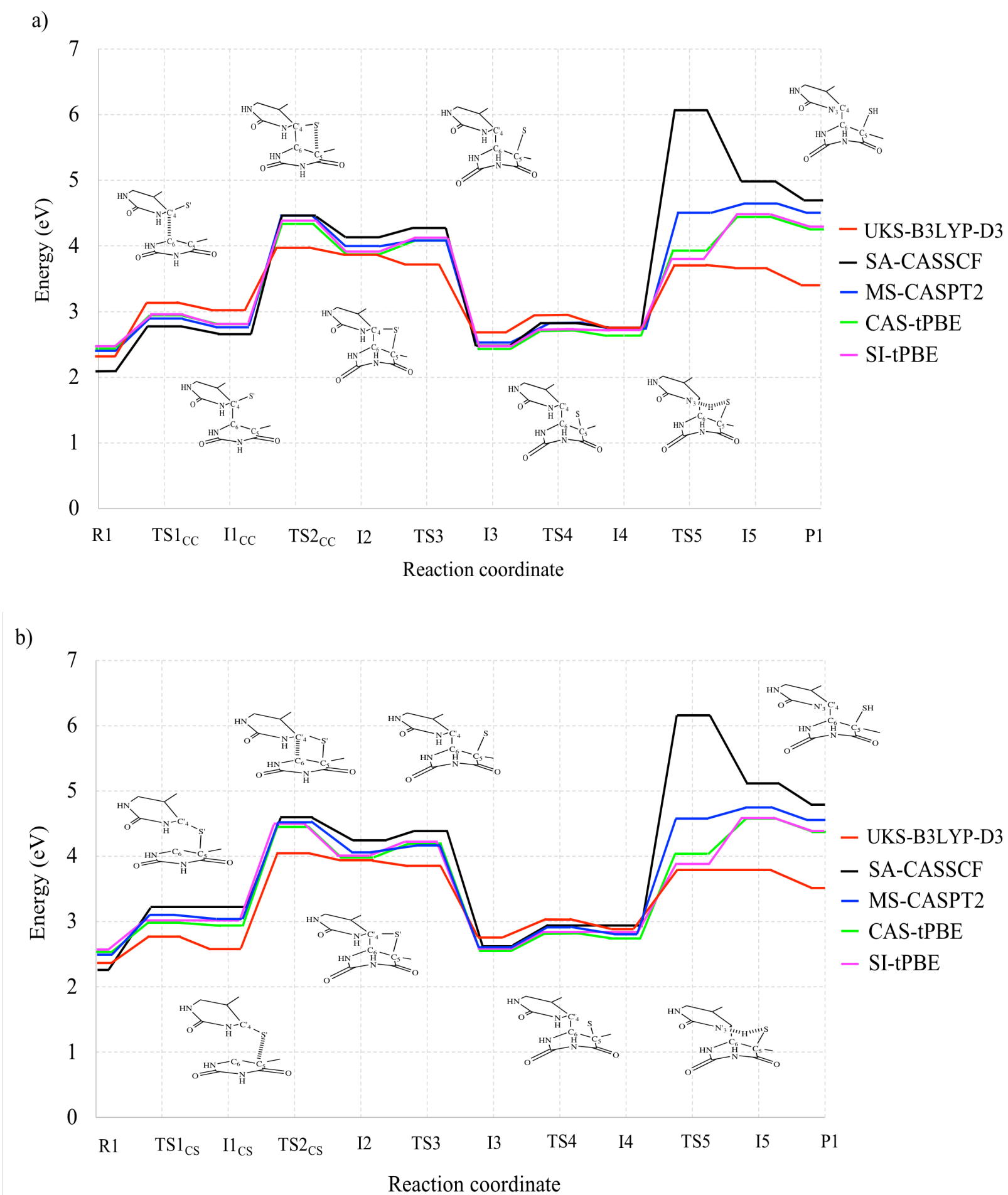

Figure S8: Potential energy profile for 6-4PP formation for the CC-CS triplet mechanism (a) and for the CS-CC triplet mechanism (b). Each reaction mechanism is calculated with UKS-B3LYP-D3/cc-pVDZ (red line), SA-CASSCF/ANO-RCC-VDZ 
(black line), MS-CASPT2/ANO-RCC-VDZ (blue line), MC-PDFT/ANO-RCC-VDZ (green line) and SI-PDFT/ANO-RCC-VDZ(pink line). The energies are in eV relative to $\mathrm{SR} 1$ in the $\mathrm{S}_{0}$ state.
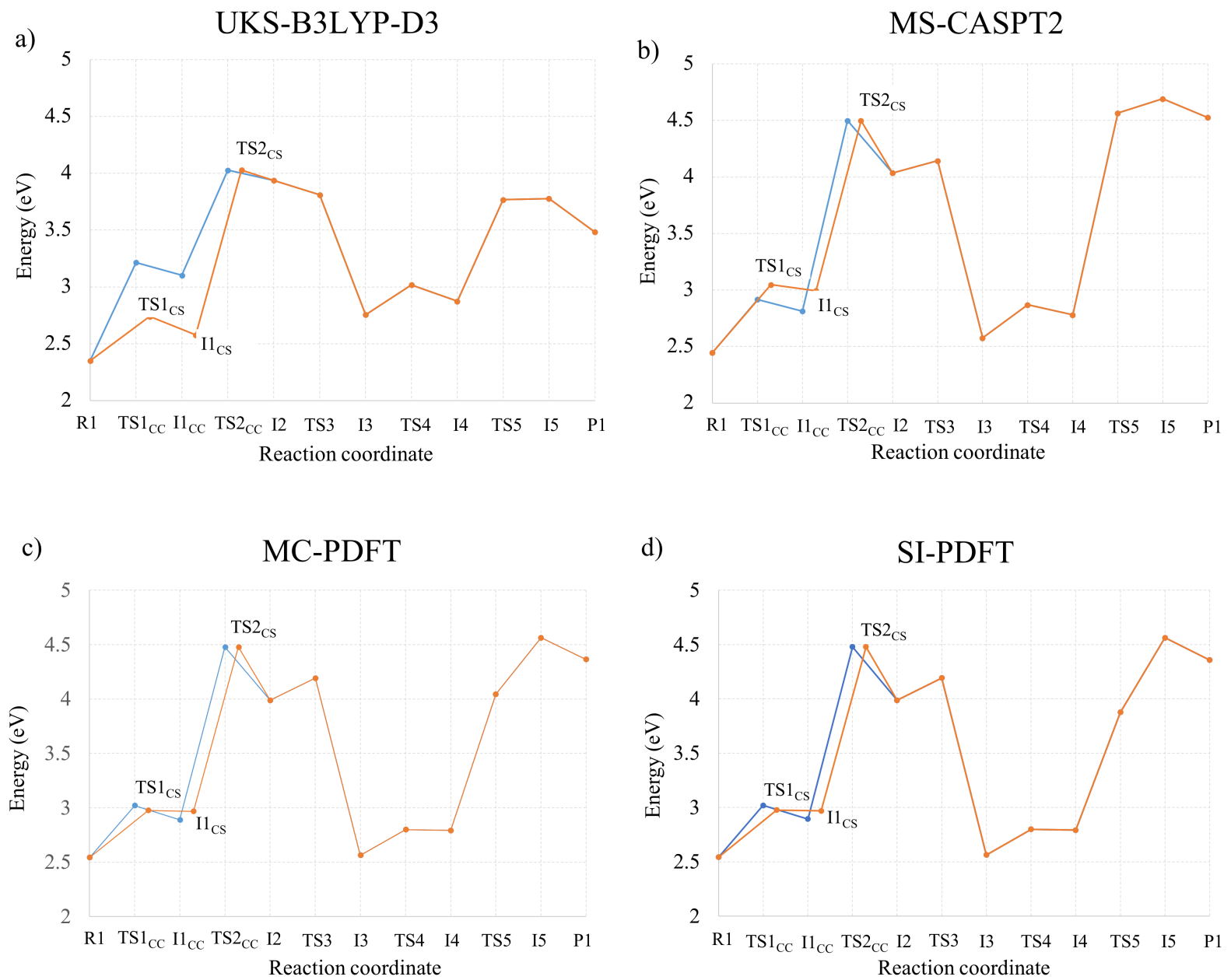

Figure S9: $\mathrm{T}_{1}$ potential energy profiles for the CC-CS (blue lines) and the CS-CC triplet mechanism (orange lines). (a) B3LYP-D3/cc-pVDZ, (b) MS-CASPT2/ANO-RCC-VDZ, (c) MC-PDFT /ANO-RCC-VDZ, and (d) SI-PDFT/ANO-RCC-VDZ. Energies are in eV relative to the $\mathrm{S}_{0}$ energy at its equilibrium geometry (SR1). 


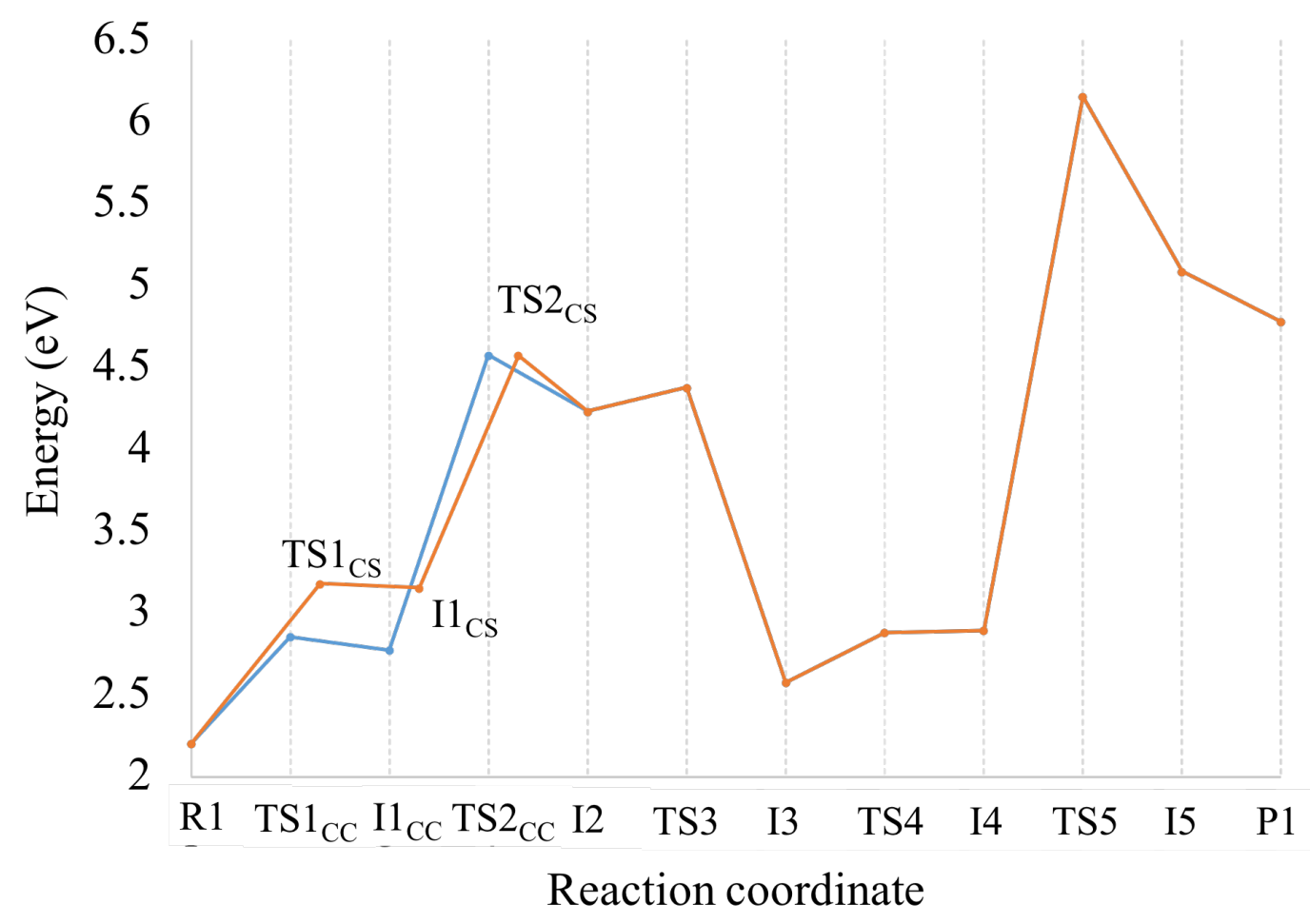

Figure 10. $\mathrm{T}_{1}$ Potential energy profiles for the CC-CS (blue line) and the CS-CC triplet mechanism (orange line) CASSCF(12,11)/ANO-RCC-VDZ. 

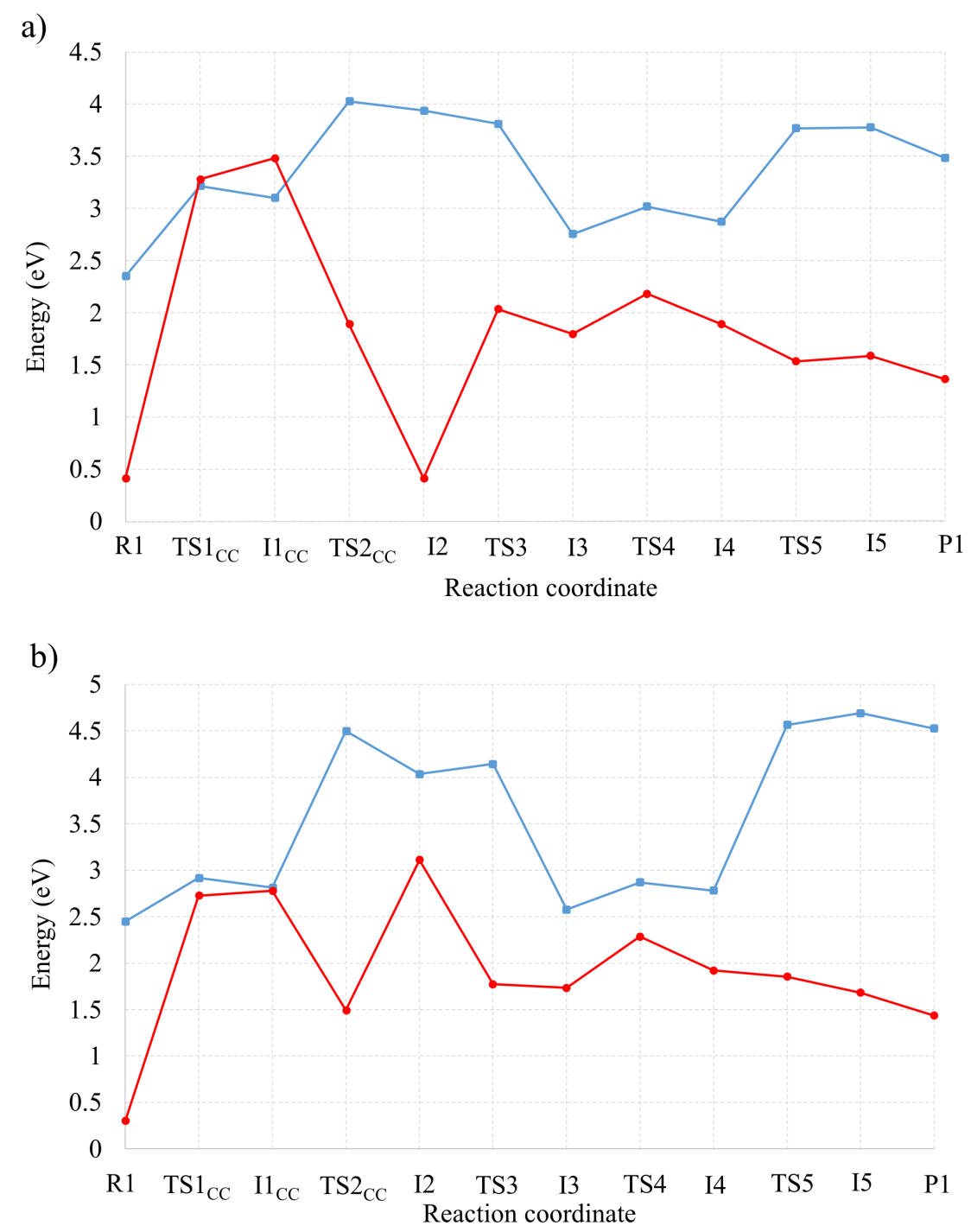

Figure S11: Potential energy profiles along the CC-CS $\mathrm{T}_{1}$ reaction path leading to 6-4PP formation computed by UKS-B3LYP-D3/cc-pVDZ level (a) and by MS-CASPT2/ANORCC-VDZ (b). The ground state $\left(\mathrm{S}_{0}\right)$ is represented by a red line and lowest triplet state $\left(\mathrm{T}_{1}\right)$ by a blue line. Energies are in $\mathrm{eV}$ relative to the $\mathrm{S}_{0}$ state at the SR1 geometry. 
a)

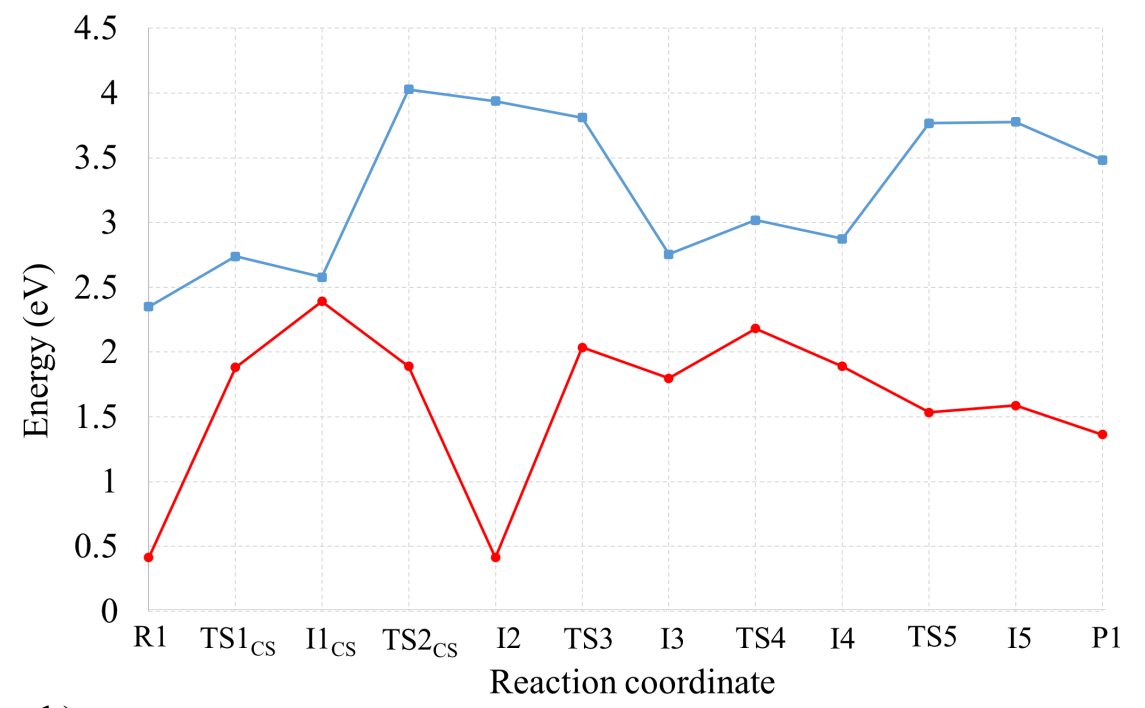

b)

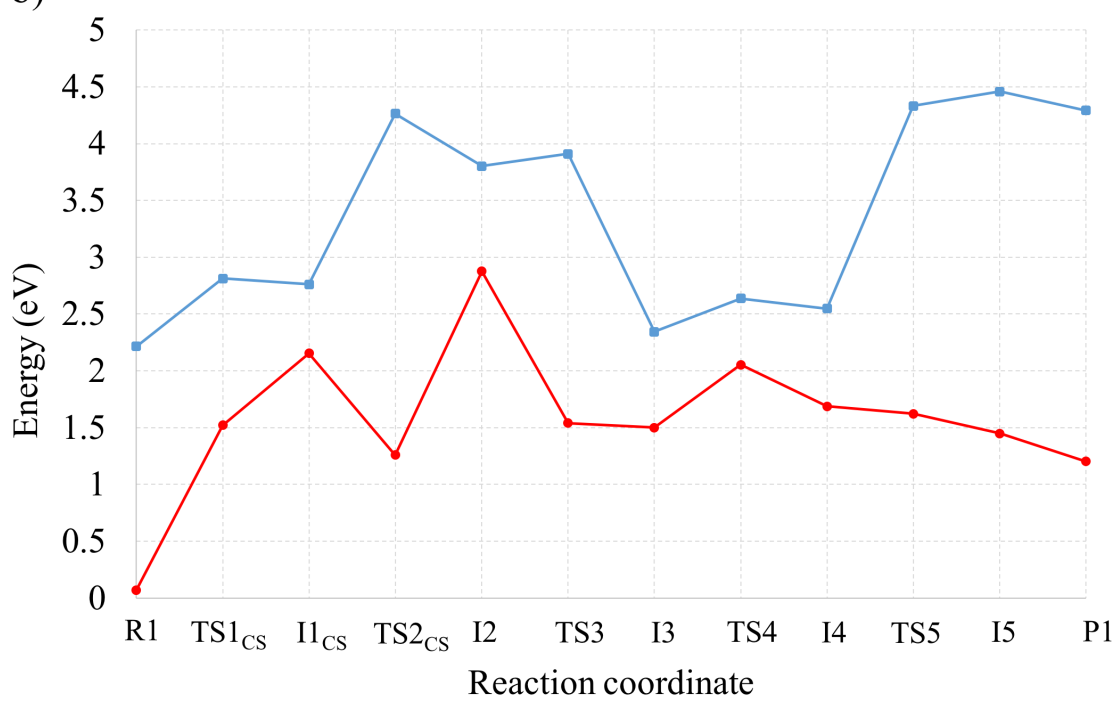

Figure S12: Potential energy profiles along the CS-CC $\mathrm{T}_{1}$ reaction path leading to 6-4PP formation computed by UKS-B3LYP-D3/cc-pVDZ (a) and by MS-CASPT2/ANO-RCCVDZ (b). The ground state $\left(\mathrm{S}_{0}\right)$ is represented by a red line and lowest triplet excited state $\left(\mathrm{T}_{1}\right)$ by a blue line. Energies are in $\mathrm{eV}$ relative to the $\mathrm{S}_{0}$ state at the $\mathrm{SR} 1$ geometry. 
Table S19. Cartesian coordinates (in $\AA$ ) for the B3LYP-D3/cc-pVDZ optimized geometries of the $\mathrm{S}_{0}$ state.

SR1 minimum

$\begin{array}{lrrr}\mathrm{H} & 2.868386 & -3.661360 & -1.883709 \\ \mathrm{C} & 2.513415 & -2.625606 & -1.788940 \\ \mathrm{O} & 1.128007 & -2.611049 & -2.152723 \\ \mathrm{C} & 0.608075 & -1.434845 & -1.532311 \\ \mathrm{C} & 2.622076 & -2.039134 & -0.358648 \\ \mathrm{C} & 1.232052 & -1.417459 & -0.142085 \\ \mathrm{O} & 3.686307 & -1.099792 & -0.334092 \\ \mathrm{H} & 3.102635 & -1.975062 & -2.461872 \\ \mathrm{H} & 0.879996 & -0.536741 & -2.104775 \\ \mathrm{H} & 2.807849 & -2.819550 & 0.394902 \\ \mathrm{H} & 0.647302 & -2.005451 & 0.577742 \\ \mathrm{H} & 1.327545 & -0.404631 & 0.240721 \\ \mathrm{~N} & -0.851085 & -1.478908 & -1.545167 \\ \mathrm{C} & -1.540421 & -0.488435 & -2.246867 \\ \mathrm{O} & -1.010944 & 0.327528 & -2.984022 \\ \mathrm{~N} & -2.907341 & -0.486192 & -2.013607 \\ \mathrm{C} & -3.632498 & -1.296754 & -1.135088 \\ \mathrm{C} & -2.859781 & -2.389401 & -0.551115 \\ \mathrm{C} & -3.585799 & -3.360220 & 0.329175 \\ \mathrm{C} & -1.522966 & -2.407442 & -0.772283 \\ \mathrm{H} & -3.384074 & 0.346521 & -2.353740 \\ \mathrm{H} & -4.305166 & -3.963382 & -0.251947 \\ \mathrm{H} & -4.157814 & -2.805806 & 1.089215 \\ \mathrm{H} & -2.886376 & -4.037381 & 0.841626 \\ \mathrm{H} & -0.880871 & -3.169141 & -0.331329 \\ \mathrm{P} & 3.909994 & -0.217089 & 1.115503 \\ \mathrm{O} & 3.138327 & -0.917634 & 2.216196 \\ \mathrm{O} & 5.347206 & 0.236268 & 1.114386 \\ \mathrm{O} & 2.928595 & 1.153161 & 0.733542 \\ \mathrm{C} & 3.330450 & 1.887577 & -0.405011 \\ \mathrm{C} & 2.198496 & 2.785320 & -0.869458 \\ \mathrm{O} & 1.055788 & 1.965907 & -1.214602 \\ \mathrm{C} & -0.151950 & 2.610667 & -0.893733 \\ \mathrm{C} & 1.661227 & 3.812036 & 0.130324 \\ \mathrm{C} & 0.220547 & 4.007170 & -0.362027 \\ \mathrm{H} & 2.249400 & 4.740968 & 0.122693 \\ \mathrm{H} & 3.597050 & 1.201718 & -1.226892 \\ \mathrm{H} & 4.219901 & 2.508289 & -0.185309 \\ \mathrm{H} & 2.534806 & 3.304627 & -1.790016 \\ \mathrm{H} & -0.815820 & 2.632040 & -1.764386 \\ \mathrm{H} & -0.483862 & 4.351095 & 0.408078\end{array}$




$\begin{array}{crrr}\mathrm{H} & 0.187793 & 4.724387 & -1.198424 \\ \mathrm{~N} & -0.890589 & 1.804521 & 0.114962 \\ \mathrm{C} & -2.269520 & 1.714706 & -0.023565 \\ \mathrm{O} & -2.907615 & 2.291960 & -0.897055 \\ \mathrm{~N} & -2.872565 & 0.890195 & 0.909866 \\ \mathrm{C} & -2.283215 & 0.231988 & 1.979138 \\ \mathrm{C} & -0.858663 & 0.424788 & 2.099173 \\ \mathrm{C} & -0.074883 & -0.226027 & 3.201879 \\ \mathrm{C} & -0.232665 & 1.187926 & 1.155905 \\ \mathrm{H} & -3.868902 & 0.732395 & 0.763835 \\ \mathrm{H} & -0.290137 & 0.265004 & 4.167070 \\ \mathrm{H} & 1.007954 & -0.202949 & 2.997579 \\ \mathrm{H} & -0.391026 & -1.274623 & 3.314794 \\ \mathrm{H} & 0.853183 & 1.306425 & 1.159652 \\ \mathrm{O} & -4.811160 & -1.053967 & -0.896747 \\ \mathrm{~S} & -3.232870 & -0.703644 & 2.996792\end{array}$

STS1 transition state
H 2.153562
$-3.375731$
$-1.983963$
C 1.975323
$-2.296489$
$-2.104777$
O 0.663708
$-2.117718$
$-2.657725$
C $\quad 0.031931$
$-0.964758$
$-2.077062$
$\begin{array}{lll}\text { C } & 1.959094 & -1.530601\end{array}$
$-0.787965$
C 1.074768
$-0.338289$
$-1.166911$
O 3.246208
$-1.180901$
$-0.325284$
H 2.750868
$-1.877326$
$-2.775039$
H -0.297162
$-0.316896$
$-2.897094$
H 1.461565
$-2.143169$
$-0.019183$
H 0.676201
0.179650
$-0.297156$
H 1.672293
0.388327
$-1.733558$
$\mathrm{N} \quad-1.201614$
$-1.367122$
$-1.375667$
C -2.280589
$-1.635867$
$-2.185093$
O -2.326417
$-1.422528$
$-3.388694$
N -3.378541
$-2.221243$
$-1.533649$
C -3.432085
$-2.719548$
$-0.242393$
C -2.335132
$-2.232982$
0.640942
C -2.126218
$-3.014160$
1.902884
C -1.251951
$-1.525450$
0.037656
H -4.118470
$-2.520057$
$-2.161775$
H -1.639598
$-3.973813$
1.643992
H -3.087625
$-3.231625$
2.382699
H -1.468356
$-2.480246$
2.595550
H -0.283482
$-1.580016$
0.529314
P 3.269526
$-0.575856$
1.269867
O 2.102130
$-1.196232$
2.022960
O 4.718096
$-0.520417$
1.680774
O 2.743735
1.025156
0.958163
C 3.617931
1.831438
0.198681 


$\begin{array}{lrrr}\mathrm{C} & 2.934154 & 3.157545 & -0.084280 \\ \mathrm{O} & 1.771519 & 2.944486 & -0.920408 \\ \mathrm{C} & 0.607310 & 3.521175 & -0.373145 \\ \mathrm{C} & 2.437209 & 3.900285 & 1.168966 \\ \mathrm{C} & 1.077467 & 4.470117 & 0.740646 \\ \mathrm{H} & 3.140056 & 4.685690 & 1.480574 \\ \mathrm{H} & 3.870450 & 1.340592 & -0.758494 \\ \mathrm{H} & 4.567110 & 2.006424 & 0.740984 \\ \mathrm{H} & 3.633029 & 3.791939 & -0.661927 \\ \mathrm{H} & 0.068404 & 4.033470 & -1.177320 \\ \mathrm{H} & 2.340901 & 3.186169 & 1.998446 \\ \mathrm{H} & 0.347044 & 4.555305 & 1.557778 \\ \mathrm{H} & 1.201252 & 5.471637 & 0.300017 \\ \mathrm{~N} & -0.343411 & 2.486117 & 0.081575 \\ \mathrm{C} & -1.384943 & 2.158153 & -0.785050 \\ \mathrm{O} & -1.613011 & 2.731454 & -1.838765 \\ \mathrm{~N} & -2.203833 & 1.141630 & -0.307282 \\ \mathrm{C} & -2.017234 & 0.336404 & 0.802878 \\ \mathrm{C} & -0.964219 & 0.777564 & 1.689333 \\ \mathrm{C} & -0.654811 & 0.062587 & 2.971339 \\ \mathrm{C} & -0.173452 & 1.807666 & 1.274089 \\ \mathrm{H} & -3.018052 & 0.949684 & -0.884314 \\ \mathrm{H} & -1.565249 & -0.397581 & 3.377909 \\ \mathrm{H} & -0.246615 & 0.763142 & 3.715711 \\ \mathrm{H} & 0.134730 & -0.690167 & 2.795709 \\ \mathrm{H} & 0.678576 & 2.114334 & 1.871016 \\ \mathrm{O} & -4.296358 & -3.512040 & 0.100273 \\ \mathrm{~S} & -3.611093 & -0.339751 & 1.412143\end{array}$

SI2 minimum
$\begin{array}{llll}\mathrm{H} & 1.885299 & -3.569902 & 0.265594\end{array}$
C $\quad 1.806811 \quad-2.916448 \quad-0.615729$
$\begin{array}{llll}\text { O } & 0.494588 & -3.077910 & -1.172558\end{array}$
C $\quad 0.081554 \quad-1.852997 \quad-1.788013$
$\begin{array}{llll}\text { C } & 1.944799 & -1.430686 & -0.280082\end{array}$
$\begin{array}{llll}\text { C } & 1.121986 & -0.784278 & -1.407445\end{array}$
$\begin{array}{llll}\text { O } & 3.299282 & -1.039721 & -0.271039\end{array}$
H $2.584909 \quad-3.201878 \quad-1.349083$

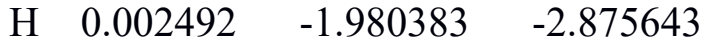
$\mathrm{H} \quad 1.492061 \quad-1.231837 \quad 0.700865$
$\mathrm{H} \quad 0.673430 \quad 0.176576 \quad-1.133204$
$\begin{array}{llll}\mathrm{H} & 1.799356 & -0.607557 & -2.256793\end{array}$
$\begin{array}{llll}\mathrm{N} & -1.296159 & -1.594652 & -1.347735\end{array}$
$\begin{array}{llll}\text { C } & -2.279328 & -1.451272 & -2.287007\end{array}$
$\begin{array}{llll}\mathrm{O} & -2.118832 & -1.369284 & -3.493526\end{array}$
$\begin{array}{llll}\mathrm{N} & -3.599262 & -1.414432 & -1.758525\end{array}$
$\begin{array}{llll}\text { C } & -4.018928 & -2.066034 & -0.600390\end{array}$
$\begin{array}{llll}\text { C } & -2.949698 & -2.184521 & 0.474371\end{array}$ 


$\begin{array}{cccc}\mathrm{C} & -2.884237 & -3.602558 & 1.017863 \\ \mathrm{C} & -1.604093 & -1.555550 & 0.062648 \\ \mathrm{H} & -4.298667 & -1.378393 & -2.497525 \\ \mathrm{H} & -2.518798 & -4.284973 & 0.231442 \\ \mathrm{H} & -3.882282 & -3.936783 & 1.334556 \\ \mathrm{H} & -2.192027 & -3.647630 & 1.871040 \\ \mathrm{H} & -0.799445 & -2.090465 & 0.575448 \\ \mathrm{P} & 3.819071 & -0.077236 & 1.037925 \\ \mathrm{O} & 3.116104 & -0.547638 & 2.299741 \\ \mathrm{O} & 5.298512 & 0.139569 & 0.834177 \\ \mathrm{O} & 2.996540 & 1.356958 & 0.635527 \\ \mathrm{C} & 3.600006 & 2.242870 & -0.279571 \\ \mathrm{C} & 2.708346 & 3.471048 & -0.370326 \\ \mathrm{O} & 1.466888 & 3.108504 & -1.008257 \\ \mathrm{C} & 0.334207 & 3.523986 & -0.265348 \\ \mathrm{C} & 2.338962 & 4.057778 & 1.004554 \\ \mathrm{C} & 0.856764 & 4.430058 & 0.864419 \\ \mathrm{H} & 2.970925 & 4.919785 & 1.262702 \\ \mathrm{H} & 3.698785 & 1.780342 & -1.278840 \\ \mathrm{H} & 4.613804 & 2.525891 & 0.059651 \\ \mathrm{H} & 3.203322 & 4.227325 & -1.010597 \\ \mathrm{H} & -0.349772 & 4.051124 & -0.939317 \\ \mathrm{H} & 2.489513 & 3.283661 & 1.769190 \\ \mathrm{H} & 0.280471 & 4.300004 & 1.791340 \\ \mathrm{H} & 0.743425 & 5.478183 & 0.544141 \\ \mathrm{~N} & -0.450084 & 2.372310 & 0.185558 \\ \mathrm{C} & -1.618997 & 2.083516 & -0.512314 \\ \mathrm{O} & -2.118389 & 2.827983 & -1.349146 \\ \mathrm{~N} & -2.201988 & 0.878591 & -0.193801 \\ \mathrm{C} & -1.756885 & -0.126949 & 0.718442 \\ \mathrm{C} & -0.586110 & 0.340361 & 1.509566 \\ \mathrm{C} & -0.062378 & -0.496435 & 2.645755 \\ \mathrm{C} & 0.016142 & 1.496794 & 1.166432 \\ \mathrm{H} & -3.043279 & 0.696480 & -0.729635 \\ \mathrm{H} & -0.622877 & -0.289468 & 3.575139 \\ \mathrm{H} & 1.014478 & -0.320956 & 2.799935 \\ \mathrm{H} & -0.186910 & -1.573813 & 2.450043 \\ \mathrm{H} & 0.961109 & 1.772403 & 1.628451 \\ \mathrm{O} & -5.157768 & -2.478949 & -0.483747 \\ \mathrm{~S} & -3.249196 & -0.841833 & 1.747984\end{array}$

STS2 transition state
H $\quad 2.297479 \quad-3.297102$
1.184886
C $\quad 2.100550 \quad-2.940166$
0.164252
$\begin{array}{lll}\text { O } & 0.725198 & -3.220865\end{array}$
$-0.136994$
$\begin{array}{llll}\text { C } & 0.352709 & -2.382270 & -1.235711\end{array}$
$\begin{array}{llll}\text { C } & 2.187700 & -1.425742 & 0.011643\end{array}$ 


\begin{tabular}{|c|c|c|c|}
\hline $\mathrm{C}$ & 1.332260 & -1.188652 & -1.236330 \\
\hline $\mathrm{O}$ & 3.504948 & -0.908065 & -0.051206 \\
\hline & 2.780058 & -3.459196 & -0.540253 \\
\hline $\mathrm{H}$ & 0.436297 & -2.944372 & -2.177087 \\
\hline H & 1.688856 & -0.993474 & 0.878624 \\
\hline 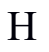 & 0.815119 & -0.223733 & -1.217739 \\
\hline $\mathrm{H}$ & 1.976966 & -1.215808 & -2.127624 \\
\hline $\mathrm{N}$ & -1.079510 & -2.066098 & -1.117578 \\
\hline $\mathrm{C}$ & -1.673324 & -1.706437 & -2.310344 \\
\hline $\mathrm{O}$ & -1.061051 & -1.608353 & -3.366180 \\
\hline $\mathrm{N}$ & -3.054859 & -1.528752 & -2.245228 \\
\hline $\mathrm{C}$ & -3.816481 & -2.206023 & -1.270193 \\
\hline $\mathrm{C}$ & -3.287853 & -2.038946 & 0.142236 \\
\hline $\mathrm{C}$ & -3.602687 & -3.284629 & 0.971440 \\
\hline $\mathrm{C}$ & -1.726129 & -1.785641 & 0.179857 \\
\hline $\mathrm{H}$ & -3.478970 & -1.510295 & -3.169892 \\
\hline $\mathrm{H}$ & -3.012881 & -4.148631 & 0.612846 \\
\hline $\mathrm{H}$ & -4.670398 & -3.521626 & 0.880299 \\
\hline $\mathrm{H}$ & -3.361900 & -3.097428 & 2.028709 \\
\hline $\mathrm{H}$ & -1.302629 & -2.507300 & 0.881137 \\
\hline$P$ & 3.629998 & 0.591048 & 0.731977 \\
\hline $\mathrm{O}$ & 3.076233 & 0.447389 & 2.151390 \\
\hline $\mathrm{O}$ & 4.942636 & 1.233803 & 0.354039 \\
\hline $\mathrm{O}$ & 2.367611 & 1.396734 & -0.078706 \\
\hline $\mathrm{C}$ & 2.644901 & 2.276179 & -1.153835 \\
\hline $\mathrm{C}$ & 2.150922 & 3.662197 & -0.775159 \\
\hline $\mathrm{O}$ & 0.717011 & 3.669579 & -0.604774 \\
\hline $\mathrm{C}$ & 0.378750 & 3.467823 & 0.746277 \\
\hline $\mathrm{C}$ & 2.697385 & 4.170792 & 0.566410 \\
\hline $\mathrm{C}$ & 1.643477 & 3.708172 & 1.594987 \\
\hline $\mathrm{H}$ & 2.764580 & 5.269399 & 0.545698 \\
\hline $\mathrm{H}$ & 2.106344 & 1.926675 & -2.052962 \\
\hline $\mathrm{H}$ & 3.728368 & 2.302233 & -1.358481 \\
\hline $\mathrm{H}$ & 2.356003 & 4.358150 & -1.605374 \\
\hline $\mathrm{H}$ & -0.435752 & 4.165908 & 0.976053 \\
\hline $\mathrm{H}$ & 3.691941 & 3.753603 & 0.781822 \\
\hline $\mathrm{H}$ & 2.008076 & 2.812107 & 2.106462 \\
\hline $\mathrm{H}$ & 1.437832 & 4.475045 & 2.355910 \\
\hline $\mathrm{N}$ & -0.261452 & 2.113276 & 0.891590 \\
\hline $\mathrm{C}$ & -1.472699 & 1.957904 & 0.158682 \\
\hline $\mathrm{O}$ & -2.113388 & 2.902336 & -0.252891 \\
\hline $\mathrm{N}$ & -1.825826 & 0.635437 & -0.048893 \\
\hline $\mathrm{C}$ & -1.296219 & -0.402568 & 0.641464 \\
\hline $\mathrm{C}$ & -0.333769 & -0.174564 & 1.624469 \\
\hline $\mathrm{C}$ & 0.160405 & -1.218823 & 2.596282 \\
\hline $\mathrm{C}$ & 0.221340 & 1.106418 & 1.635816 \\
\hline $\mathrm{H}$ & -2.901043 & 0.448733 & -0.204063 \\
\hline $\mathrm{H}$ & -0.555417 & -1.337824 & 3.427241 \\
\hline $\mathrm{H}$ & 1.139678 & -0.916405 & 2.995577 \\
\hline $\mathrm{H}$ & 0.277362 & -2.198583 & 2.115713 \\
\hline
\end{tabular}




$\begin{array}{cccc}\mathrm{H} & 1.142704 & 1.265629 & 2.199153 \\ \mathrm{O} & -4.826445 & -2.807402 & -1.582268 \\ \mathrm{~S} & -4.289672 & -0.582463 & 0.731716\end{array}$

SP1 minimum

\begin{tabular}{|c|c|c|c|}
\hline $\mathrm{H}$ & 1.909522 & -2.842456 & 1.098963 \\
\hline $\mathrm{C}$ & 1.830547 & -2.775928 & 0.007120 \\
\hline $\mathrm{O}$ & 0.571035 & -3.340623 & -0.41455 \\
\hline C & 0.055833 & -2.599551 & \\
\hline $\mathrm{C}$ & 1.822018 & -1.333097 & \\
\hline C & 1.075392 & -1.488795 & \\
\hline $\mathrm{O}$ & 3.113144 & -0.778976 & \\
\hline & 2.671983 & -3.323791 & \\
\hline & -0.101373 & -3.257085 & \\
\hline $\mathrm{H}$ & 1.2246 & -0.720075 & \\
\hline $\mathrm{H}$ & 0.591762 & -0.572929 & -2.1 \\
\hline ח & 1.782059 & 5992 & \\
\hline$\Lambda$ & -1.301252 & -2.13 & -1 . \\
\hline $\mathrm{C}$ & -2.2069 & -1.98 & \\
\hline $\mathrm{O}$ & -1.984573 & -2.198607 & \\
\hline $\mathrm{N}$ & -3.511369 & -1.627784 & \\
\hline C & -3.9749 & -1.431945 & -0. \\
\hline $\mathrm{C}$ & -3.0 & -1.9 & \\
\hline C & -3.190329 & -3.4 & \\
\hline$(\mathrm{C}$ & -1.552189 & -1.59 & 0.1 \\
\hline $\mathrm{F}$ & -4.12 & -1.3 & -2. \\
\hline $\mathrm{H}$ & -2.9511 & -3.97 & -0 . \\
\hline $\mathrm{H}$ & -4.227019 & -3.677166 & \\
\hline $\mathrm{H}$ & -2.5187 & -3.83 & \\
\hline $\mathrm{H}$ & -0.87 & -2.1 & \\
\hline $\mathrm{P}$ & 3.7063 & -0.02 & \\
\hline $\mathrm{O}$ & 3.070100 & -0.6 & \\
\hline $\mathrm{O}$ & 5.1769 & 0.2 & \\
\hline $\mathrm{O}$ & 2.869039 & 1.4 & \\
\hline $\mathrm{C}$ & 3.452971 & 2.422355 & -0.2 \\
\hline $\mathrm{C}$ & 2.638105 & 3.710146 & -0.2 \\
\hline $\mathrm{O}$ & 1.42 & 3.57 & -1.0 \\
\hline $\mathrm{C}$ & 0.272373 & 3.742774 & -0.20860 \\
\hline $\mathrm{C}$ & 2.211648 & 4.178075 & 1.17996 \\
\hline $\mathrm{C}$ & 0.722057 & 4.531626 & 1.02844 \\
\hline $\mathrm{H}$ & 2.807806 & 5.035846 & 1.52126 \\
\hline $\mathrm{H}$ & 3.496848 & 2.042419 & -1.27778 \\
\hline $\mathrm{H}$ & 4.488740 & 2.638851 & 0.07864 \\
\hline & 3.234300 & 4.482910 & -0.74756 \\
\hline $\mathrm{H}$ & -0.475676 & 4.261056 & $-0.8165 c$ \\
\hline $\mathrm{H}$ & 2.364743 & 3.353277 & 1.88923 \\
\hline $\mathrm{H}$ & 0.115383 & 4.308171 & 1.91800 \\
\hline & 0.595022 & 5.602164 & 0.80783 \\
\hline
\end{tabular}




$\begin{array}{cccc}\mathrm{N} & -0.360617 & 2.438782 & 0.089569 \\ \mathrm{C} & -1.294213 & 1.944727 & -0.876769 \\ \mathrm{O} & -1.734098 & 2.689731 & -1.742283 \\ \mathrm{~N} & -1.613507 & 0.607576 & -0.773437 \\ \mathrm{C} & -1.228546 & -0.100538 & 0.265855 \\ \mathrm{C} & -0.474445 & 0.416015 & 1.359211 \\ \mathrm{C} & -0.049133 & -0.346650 & 2.589541 \\ \mathrm{C} & -0.003736 & 1.689525 & 1.159490 \\ \mathrm{H} & -4.676193 & -0.799811 & 1.774236 \\ \mathrm{H} & -0.331292 & 0.214239 & 3.495971 \\ \mathrm{H} & 1.046735 & -0.500291 & 2.585711 \\ \mathrm{H} & -0.544874 & -1.322513 & 2.667598 \\ \mathrm{H} & 0.708713 & 2.128352 & 1.852587 \\ \mathrm{O} & -5.094402 & -0.996087 & -0.259678 \\ \mathrm{~S} & -3.423542 & -1.105683 & 2.214394\end{array}$


Table S20. Cartesian coordinates (in $\AA$ ) optimized by UKS-B3LYP-D3/aug-cc-pVDZ calculations for the lowest triplet state.

$\mathrm{R} 1$ minimum

$\begin{array}{lccc}\mathrm{H} & 2.767577 & -3.638136 & -1.639364 \\ \mathrm{C} & 2.433956 & -2.591234 & -1.617600 \\ \mathrm{O} & 1.075060 & -2.566827 & -2.060039 \\ \mathrm{C} & 0.534429 & -1.346592 & -1.553753 \\ \mathrm{C} & 2.476266 & -1.929464 & -0.218016 \\ \mathrm{C} & 1.125839 & -1.190844 & -0.151567 \\ \mathrm{O} & 3.619973 & -1.091724 & -0.164580 \\ \mathrm{H} & 3.076853 & -1.991980 & -2.289359 \\ \mathrm{H} & 0.787533 & -0.495374 & -2.201896 \\ \mathrm{H} & 2.537089 & -2.679373 & 0.586606 \\ \mathrm{H} & 0.467334 & -1.643948 & 0.601580 \\ \mathrm{H} & 1.270758 & -0.139445 & 0.098109 \\ \mathrm{~N} & -0.923081 & -1.442556 & -1.578594 \\ \mathrm{C} & -1.650752 & -0.474894 & -2.272390 \\ \mathrm{O} & -1.147884 & 0.387647 & -2.973587 \\ \mathrm{~N} & -3.021612 & -0.564227 & -2.077667 \\ \mathrm{C} & -3.717961 & -1.449195 & -1.248212 \\ \mathrm{C} & -2.889941 & -2.496774 & -0.656952 \\ \mathrm{C} & -3.578792 & -3.541525 & 0.166905 \\ \mathrm{C} & -1.549388 & -2.424140 & -0.837324 \\ \mathrm{H} & -3.538614 & 0.245909 & -2.411845 \\ \mathrm{H} & -4.184779 & -3.058579 & 0.949888 \\ \mathrm{H} & -2.855841 & -4.216973 & 0.648412 \\ \mathrm{H} & -4.269756 & -4.139435 & -0.452666 \\ \mathrm{H} & -0.866529 & -3.152288 & -0.399098 \\ \mathrm{P} & 3.859729 & -0.108158 & 1.210302 \\ \mathrm{O} & 3.009174 & -0.648674 & 2.348200 \\ \mathrm{O} & 5.328173 & 0.228187 & 1.244703 \\ \mathrm{O} & 2.996401 & 1.276739 & 0.689478 \\ \mathrm{C} & 3.363780 & 1.868764 & -0.538037 \\ \mathrm{C} & 2.244042 & 2.799292 & -0.971468 \\ \mathrm{O} & 1.053018 & 2.017917 & -1.196949 \\ \mathrm{C} & -0.113054 & 2.709559 & -0.793350 \\ \mathrm{C} & 1.838353 & 3.866242 & 0.048029 \\ \mathrm{C} & 0.355195 & 4.085408 & -0.272642 \\ \mathrm{H} & 2.443816 & 4.778874 & -0.055522 \\ \mathrm{H} & 3.515308 & 1.097170 & -1.312911 \\ \mathrm{H} & 4.307195 & 2.440452 & -0.444401 \\ \mathrm{H} & 2.532889 & 3.275577 & -1.931022 \\ \mathrm{H} & -0.813908 & 2.782489 & -1.631940 \\ \mathrm{H} & 1.979119 & 3.466201 & 1.062374 \\ & -0.244969 & 4.415475 & 0.586276 \\ & 0.832527 & 1.911998 & 0.217302\end{array}$




$\begin{array}{lrrr}\mathrm{C} & -2.197031 & 1.673308 & -0.008959 \\ \mathrm{O} & -2.820444 & 2.208764 & -0.920043 \\ \mathrm{~N} & -2.778464 & 0.783240 & 0.855188 \\ \mathrm{C} & -2.130125 & 0.082330 & 1.906250 \\ \mathrm{C} & -0.802638 & 0.420841 & 2.161933 \\ \mathrm{C} & 0.019777 & -0.197110 & 3.236800 \\ \mathrm{C} & -0.167806 & 1.427384 & 1.344517 \\ \mathrm{H} & -3.753926 & 0.569969 & 0.674003 \\ \mathrm{H} & 0.209213 & 0.554959 & 4.026097 \\ \mathrm{H} & 1.026298 & -0.474714 & 2.858858 \\ \mathrm{H} & -0.496868 & -1.053803 & 3.686184 \\ \mathrm{H} & 0.914471 & 1.539992 & 1.358767 \\ \mathrm{O} & -4.922735 & -1.311888 & -1.059231 \\ \mathrm{~S} & -3.087071 & -1.032970 & 2.811689\end{array}$

TS1 $1_{\mathrm{CC}}$ transition state

$\begin{array}{lccc}\mathrm{H} & 1.811465 & -3.727465 & -0.143266 \\ \mathrm{C} & 1.787724 & -2.910881 & -0.879686 \\ \mathrm{O} & 0.487331 & -2.897901 & -1.482945 \\ \mathrm{C} & 0.144885 & -1.553577 & -1.855432 \\ \mathrm{C} & 1.969727 & -1.522587 & -0.270778 \\ \mathrm{C} & 1.264249 & -0.653735 & -1.323874 \\ \mathrm{O} & 3.336947 & -1.228667 & -0.079329 \\ \mathrm{H} & 2.577691 & -3.085936 & -1.635120 \\ \mathrm{H} & 0.042687 & -1.480317 & -2.945333 \\ \mathrm{H} & 1.444895 & -1.466977 & 0.696161 \\ \mathrm{H} & 0.910527 & 0.313064 & -0.959794 \\ \mathrm{H} & 1.985292 & -0.462891 & -2.134105 \\ \mathrm{~N} & -1.216041 & -1.286528 & -1.362527 \\ \mathrm{C} & -2.215989 & -1.161907 & -2.306531 \\ \mathrm{O} & -2.043419 & -0.861874 & -3.476204 \\ \mathrm{~N} & -3.512433 & -1.420091 & -1.813635 \\ \mathrm{C} & -3.768931 & -2.394192 & -0.824316 \\ \mathrm{C} & -2.666416 & -2.563331 & 0.095954 \\ \mathrm{C} & -2.675821 & -3.645312 & 1.113201 \\ \mathrm{C} & -1.571866 & -1.568432 & 0.013436 \\ \mathrm{H} & -4.223598 & -1.355861 & -2.538044 \\ \mathrm{H} & -1.867383 & -4.370813 & 0.899113 \\ \mathrm{H} & -3.642390 & -4.166507 & 1.103839 \\ \mathrm{H} & -2.482184 & -3.246413 & 2.123750 \\ \mathrm{H} & -0.684578 & -1.947119 & 0.522872 \\ \mathrm{P} & 3.677134 & -0.138652 & 1.186551 \\ \mathrm{O} & 2.832880 & -0.506154 & 2.394034 \\ \mathrm{O} & 5.163934 & 0.109121 & 1.165522 \\ \mathrm{O} & 2.869532 & 1.217180 & 0.553150 \\ \mathrm{C} & 3.500644 & 2.018760 & -0.417588 \\ \mathrm{C} & 2.709809 & 3.316319 & -0.501062 \\ \mathrm{O} & 1.420605 & 3.044743 & -1.090210\end{array}$




$\begin{array}{lrrr}\mathrm{C} & 0.342738 & 3.448038 & -0.263840 \\ \mathrm{C} & 2.446305 & 3.956334 & 0.876608 \\ \mathrm{C} & 0.950082 & 4.307137 & 0.861325 \\ \mathrm{H} & 3.078047 & 4.842261 & 1.038134 \\ \mathrm{H} & 3.493016 & 1.525986 & -1.408813 \\ \mathrm{H} & 4.553457 & 2.209211 & -0.141127 \\ \mathrm{H} & 3.238805 & 4.014845 & -1.177182 \\ \mathrm{H} & -0.362253 & 4.014686 & -0.881383 \\ \mathrm{H} & 2.676207 & 3.223643 & 1.660955 \\ \mathrm{H} & 0.451157 & 4.127995 & 1.824570 \\ \mathrm{H} & 0.799008 & 5.366460 & 0.603786 \\ \mathrm{~N} & -0.452846 & 2.300419 & 0.186708 \\ \mathrm{C} & -1.670978 & 2.082944 & -0.453919 \\ \mathrm{O} & -2.176903 & 2.871111 & -1.245668 \\ \mathrm{~N} & -2.304371 & 0.907501 & -0.120763 \\ \mathrm{C} & -1.915420 & -0.099570 & 0.823575 \\ \mathrm{C} & -0.673787 & 0.309791 & 1.563476 \\ \mathrm{C} & -0.190067 & -0.535215 & 2.707643 \\ \mathrm{C} & -0.013913 & 1.419425 & 1.178145 \\ \mathrm{H} & -3.216691 & 0.810174 & -0.551803 \\ \mathrm{H} & -0.792371 & -0.357274 & 3.615236 \\ \mathrm{H} & 0.879437 & -0.354515 & 2.894897 \\ \mathrm{H} & -0.294879 & -1.611895 & 2.486102 \\ \mathrm{H} & 0.951490 & 1.654600 & 1.620368 \\ \mathrm{O} & -4.842999 & -2.992625 & -0.785246 \\ \mathrm{~S} & -3.313703 & -0.446419 & 1.973152\end{array}$

TS1 $1_{C S}$ transition state

$\begin{array}{lrrr}\mathrm{H} & 2.657737 & -3.369829 & -2.004575 \\ \mathrm{C} & 2.290230 & -2.359718 & -1.775486 \\ \mathrm{O} & 0.881165 & -2.353077 & -2.021048 \\ \mathrm{C} & 0.386126 & -1.209552 & -1.317951 \\ \mathrm{C} & 2.484792 & -1.919805 & -0.307638 \\ \mathrm{C} & 1.191641 & -1.133211 & -0.025023 \\ \mathrm{O} & 3.683453 & -1.171755 & -0.197010 \\ \mathrm{H} & 2.810238 & -1.630355 & -2.425278 \\ \mathrm{H} & 0.501631 & -0.301890 & -1.926687 \\ \mathrm{H} & 2.541487 & -2.797621 & 0.358211 \\ \mathrm{H} & 0.652891 & -1.566410 & 0.820785 \\ \mathrm{H} & 1.425103 & -0.098293 & 0.216079 \\ \mathrm{~N} & -1.058788 & -1.382007 & -1.131615 \\ \mathrm{C} & -1.920468 & -0.621328 & -1.918854 \\ \mathrm{O} & -1.554014 & 0.247018 & -2.691553 \\ \mathrm{~N} & -3.270615 & -0.945958 & -1.773770 \\ \mathrm{C} & -3.855794 & -1.880007 & -0.920866 \\ \mathrm{C} & -2.911469 & -2.476497 & 0.066890 \\ \mathrm{C} & -3.356467 & -3.798568 & 0.651354 \\ \mathrm{C} & -1.528355 & -2.329700 & -0.255610\end{array}$




$\begin{array}{lccc}\mathrm{H} & -3.892995 & -0.439848 & -2.397325 \\ \mathrm{H} & -3.341013 & -4.582923 & -0.126022 \\ \mathrm{H} & -4.385469 & -3.719555 & 1.027849 \\ \mathrm{H} & -2.692823 & -4.099917 & 1.473475 \\ \mathrm{H} & -0.771142 & -2.931162 & 0.239032 \\ \mathrm{P} & 3.937267 & -0.229483 & 1.210210 \\ \mathrm{O} & 3.065013 & -0.776396 & 2.325719 \\ \mathrm{O} & 5.413410 & 0.073875 & 1.251746 \\ \mathrm{O} & 3.111472 & 1.192270 & 0.709648 \\ \mathrm{C} & 3.520915 & 1.804907 & -0.492794 \\ \mathrm{C} & 2.493070 & 2.857260 & -0.874109 \\ \mathrm{O} & 1.238433 & 2.209719 & -1.176600 \\ \mathrm{C} & 0.132809 & 2.987705 & -0.746221 \\ \mathrm{C} & 2.169471 & 3.875202 & 0.222261 \\ \mathrm{C} & 0.718539 & 4.255741 & -0.087584 \\ \mathrm{H} & 2.856958 & 4.733559 & 0.195004 \\ \mathrm{H} & 3.600644 & 1.058941 & -1.303452 \\ \mathrm{H} & 4.514129 & 2.281761 & -0.382255 \\ \mathrm{H} & 2.843606 & 3.376829 & -1.789931 \\ \mathrm{H} & -0.515342 & 3.229177 & -1.597016 \\ \mathrm{H} & 2.265115 & 3.383250 & 1.200817 \\ \mathrm{H} & 0.132801 & 4.555509 & 0.792332 \\ \mathrm{H} & 0.676611 & 5.080899 & -0.817251 \\ \mathrm{~N} & -0.723812 & 2.181986 & 0.132458 \\ \mathrm{C} & -2.080390 & 2.126836 & -0.180186 \\ \mathrm{O} & -2.635140 & 2.883522 & -0.967550 \\ \mathrm{~N} & -2.762186 & 1.127683 & 0.477005 \\ \mathrm{C} & -2.239074 & 0.224737 & 1.424282 \\ \mathrm{C} & -0.916090 & 0.454647 & 1.844949 \\ \mathrm{C} & -0.266980 & -0.329256 & 2.955042 \\ \mathrm{C} & -0.190325 & 1.442226 & 1.194201 \\ \mathrm{H} & -3.747351 & 1.064437 & 0.247114 \\ \mathrm{H} & -0.662255 & -1.355767 & 2.979952 \\ \mathrm{H} & -0.508465 & 0.127350 & 3.931539 \\ \mathrm{H} & 0.830111 & -0.367110 & 2.842366 \\ \mathrm{~S} & 0.866691 & 1.598431 & 1.400754 \\ & -5.041642 & -2.156072 & -1.011711 \\ & -3.282647 & -1.120785 & 1.828822\end{array}$

I1 ${ }_{\mathrm{CC}}$ minimum

$\begin{array}{llll}\mathrm{H} & 1.880131 & -3.682679 & 0.114486 \\ \mathrm{C} & 1.829704 & -2.936023 & -0.691192 \\ \mathrm{O} & 0.510563 & -2.981330 & -1.251529 \\ \mathrm{C} & 0.179262 & -1.689880 & -1.779922 \\ \mathrm{C} & 2.021067 & -1.496540 & -0.218827 \\ \mathrm{C} & 1.289260 & -0.730034 & -1.330871 \\ \mathrm{O} & 3.390712 & -1.184680 & -0.093078 \\ \mathrm{H} & 2.596333 & -3.177247 & -1.452714\end{array}$




\begin{tabular}{|c|c|c|c|}
\hline & & & \\
\hline & 1.516533 & -1.356486 & 0.746607 \\
\hline & 0.914838 & 0.254377 & -1.037186 \\
\hline & 2.000158 & -0.587639 & -2.159478 \\
\hline & -1.191672 & -1.369549 & -1.348287 \\
\hline & -2.123608 & -1.145440 & -2.33496 \\
\hline & -1.864102 & -0.861362 & -3.493491 \\
\hline & -3.467886 & -1.271639 & -1.5 \\
\hline & -3.866729 & -2.230692 & -0.951890 \\
\hline & -2.851458 & -2.487802 & \\
\hline & -3.00 & -3.592 & \\
\hline & -1.629 & $-1.61 c$ & \\
\hline & -4.12 & -1.154712 & -2.6 \\
\hline & -2.20 & -4.34 & 0.8 \\
\hline & -3.9 & $-4.0^{\prime}$ & \\
\hline & -2.914079 & -3.223638 & 2.0 \\
\hline & -0.81 & -2.162994 & 0.5 \\
\hline & 3.79 & -0.08 & \\
\hline & 3.06 & -0.4 & \\
\hline & 5.27 & 0.181723 & \\
\hline & 2.91 & 1.2 & \\
\hline & 3.43 & & \\
\hline & 2.58 & & \\
\hline & 1.28 & & \\
\hline & 0.22 & 3.4 & \\
\hline & 2.3 & & \\
\hline & 0.8 & & \\
\hline & 2.9 & & \\
\hline & 3.3 & & \\
\hline & 4.45 & 2.25 & -0.2 \\
\hline & 3.06 & & \\
\hline & -0.5 & 3.951 & -0.8 \\
\hline & 2.5 & 3.23 & \\
\hline & & & \\
\hline & 0.68 & 5.356 & 0.5 \\
\hline & -0.5 & 2.25 & \\
\hline & -1.7 & 2.0 & 12 \\
\hline & -2.3 & & \\
\hline & -2.3 & 0.796 & $-0 .($ \\
\hline & -1.82 & -0.2 & \\
\hline & -0.57 & & \\
\hline & -0.02 & -0.673259 & \\
\hline & -0.00 & & \\
\hline & -3.182902 & 0.595228 & -0.5 \\
\hline & -0.676316 & -0.595093 & \\
\hline & 1.01 & -0.407609 & 2.854617 \\
\hline & -0.022592 & -1.741072 & \\
\hline & & & 1.611805 \\
\hline & -4.979203 & -2.751489 & -1.005220 \\
\hline & -3.246820 & -0.356714 & 2.067610 \\
\hline
\end{tabular}


I1 $1_{\mathrm{CS}}$ minimum

\begin{tabular}{|c|c|c|c|}
\hline & 1.900028 & -3.181857 & \\
\hline & 2.032426 & -2.845734 & -0.222086 \\
\hline & 0.863218 & -3.232725 & 988326 \\
\hline & 0.411445 & -2.155146 & -1.772669 \\
\hline & 2.153015 & -1.326357 & -0.357586 \\
\hline & 20 & & 813 \\
\hline & 3.4960 & & 207 \\
\hline & 2.926405 & 10 & -0.653051 \\
\hline & 0.2306 & 88 & 0797 \\
\hline & & & \\
\hline & 1.08 & -0.0 & -1.8 \\
\hline & 2.208 & -1.2 & -2.501262 \\
\hline & -0.9 & -1. & -1.2 \\
\hline & & -0. & \\
\hline & -1.0572 & -0.1 & -2.971158 \\
\hline & -2.901752 & -0.4 & -1.661933 \\
\hline & -3.71 & & -0.8 \\
\hline & -3.0 & & \\
\hline & -3.83 & -3.4 & 0.2 \\
\hline & -1.60 & -2.4 & \\
\hline & -3.3 & & \\
\hline & -3.8 & -4. & -0.715039 \\
\hline & -4.8 & -3.2 & 0.569149 \\
\hline & -3.3 & -4 & \\
\hline & -1.0 & -3.2 & 0.1 \\
\hline & & & \\
\hline & 3.3 & -0.5 & 2.2 \\
\hline & 5.4 & & \\
\hline 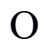 & 3.0 & & 0.67 \\
\hline & 3.36 & & \\
\hline( & 2.22 & & -0.781319 \\
\hline 0 & $1.0^{\circ}$ & & -1.2 \\
\hline $\mathrm{C}$ & & & -0.7 \\
\hline $\mathrm{C}$ & & & \\
\hline $\mathrm{C}$ & 0.2 & 4.2 & \\
\hline $\mathrm{H}$ & 2.30 & 4.8 & 0.493 \\
\hline $\mathrm{H}$ & & & -1.328080 \\
\hline $\mathrm{H}$ & 4.28 & 2.7 & -0.267666 \\
\hline & 2.54 & & -1.626 \\
\hline $\mathrm{H}$ & -0.834750 & & -1.557303 \\
\hline 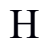 & & 3.403859 & 1.312772 \\
\hline & -0.42 & & \\
\hline & & 5.048329 & -0.768467 \\
\hline $\mathrm{N}$ & -0.803817 & & 0.168414 \\
\hline & -2.190852 & 1.961003 & 0.128657 \\
\hline & -2.851579 & 2.698005 & -0.600188 \\
\hline
\end{tabular}




$\begin{array}{lrrl}\mathrm{N} & -2.783316 & 1.062816 & 0.988486 \\ \mathrm{C} & -2.107542 & 0.024742 & 1.663227 \\ \mathrm{C} & -0.702248 & 0.139779 & 1.773360 \\ \mathrm{C} & 0.101533 & -0.788314 & 2.646892 \\ \mathrm{C} & -0.082283 & 1.133300 & 1.045050 \\ \mathrm{H} & -3.790581 & 0.992784 & 0.886713 \\ \mathrm{H} & -0.095438 & -1.838925 & 2.378567 \\ \mathrm{H} & -0.201952 & -0.673485 & 3.701333 \\ \mathrm{H} & 1.184814 & -0.601697 & 2.570779 \\ \mathrm{H} & 0.999495 & 1.264390 & 1.043271 \\ \mathrm{O} & -4.918953 & -1.011977 & -0.769508 \\ \mathrm{~S} & -3.048715 & -1.424062 & 1.940229\end{array}$

$\mathrm{TS} 2_{\mathrm{CC}}$ transition state

$\begin{array}{lccc}\mathrm{H} & 1.659318 & -3.273653 & 0.386475 \\ \mathrm{C} & 1.708029 & -2.758269 & -0.584776 \\ \mathrm{O} & 0.460142 & -2.953462 & -1.271586 \\ \mathrm{C} & 0.043609 & -1.741573 & -1.899605 \\ \mathrm{C} & 1.892486 & -1.246396 & -0.446840 \\ \mathrm{C} & 1.172472 & -0.725587 & -1.690452 \\ \mathrm{O} & 3.261012 & -0.899148 & -0.386210 \\ \mathrm{H} & 2.545118 & -3.174784 & -1.174064 \\ \mathrm{H} & -0.148109 & -1.917912 & -2.965453 \\ \mathrm{H} & 1.376598 & -0.897957 & 0.457811 \\ \mathrm{H} & 0.817792 & 0.307455 & -1.595183 \\ \mathrm{H} & 1.866320 & -0.776681 & -2.545375 \\ \mathrm{~N} & -1.279066 & -1.373077 & -1.350440 \\ \mathrm{C} & -2.282326 & -1.094958 & -2.250622 \\ \mathrm{O} & -2.102183 & -0.807408 & -3.425974 \\ \mathrm{~N} & -3.583160 & -1.184697 & -1.716530 \\ \mathrm{C} & -3.947116 & -2.045453 & -0.658337 \\ \mathrm{C} & -2.887890 & -2.251101 & 0.357384 \\ \mathrm{C} & -2.812044 & -3.674400 & 0.859722 \\ \mathrm{C} & -1.581188 & -1.528418 & 0.048461 \\ \mathrm{H} & -4.301932 & -1.073930 & -2.428037 \\ \mathrm{H} & -2.321458 & -4.312531 & 0.102187 \\ \mathrm{H} & -3.826136 & -4.060932 & 1.035260 \\ \mathrm{H} & -2.231422 & -3.731621 & 1.792222 \\ \mathrm{H} & -0.752898 & -2.065911 & 0.518077 \\ \mathrm{P} & 3.797102 & -0.217091 & 1.079712 \\ \mathrm{O} & 2.940347 & -0.789358 & 2.198578 \\ \mathrm{O} & 5.300295 & -0.144203 & 1.007356 \\ \mathrm{O} & 3.159894 & 1.353270 & 0.866752 \\ \mathrm{C} & 3.703550 & 2.156613 & -0.159190 \\ \mathrm{C} & 2.740883 & 3.303392 & -0.421387 \\ \mathrm{O} & 1.544226 & 2.785134 & -1.049624 \\ \mathrm{C} & 0.371033 & 3.318236 & -0.472168 \\ \mathrm{C} & 2.267993 & 4.033654 & 0.843420\end{array}$




$\begin{array}{rrrr}\mathrm{C} & 0.821947 & 4.414862 & 0.510444 \\ \mathrm{H} & 2.902624 & 4.902716 & 1.073181 \\ \mathrm{H} & 3.839104 & 1.574552 & -1.086917 \\ \mathrm{H} & 4.690720 & 2.559413 & 0.137399 \\ \mathrm{H} & 3.209211 & 4.010753 & -1.133966 \\ \mathrm{H} & -0.288837 & 3.704688 & -1.255897 \\ \mathrm{H} & 2.312377 & 3.339529 & 1.693936 \\ \mathrm{H} & 0.163186 & 4.476098 & 1.387736 \\ \mathrm{H} & 0.783837 & 5.383074 & -0.015784 \\ \mathrm{~N} & -0.422176 & 2.236508 & 0.147901 \\ \mathrm{C} & -1.721444 & 2.030313 & -0.376760 \\ \mathrm{O} & -2.237943 & 2.819677 & -1.161048 \\ \mathrm{~N} & -2.349779 & 0.904608 & 0.060014 \\ \mathrm{C} & -1.820007 & -0.154146 & 0.893401 \\ \mathrm{C} & -0.670996 & 0.330899 & 1.674979 \\ \mathrm{C} & -0.086546 & -0.397005 & 2.827280 \\ \mathrm{C} & 0.111477 & 1.429490 & 1.115601 \\ \mathrm{H} & -3.246045 & 0.738007 & -0.382495 \\ \mathrm{H} & -0.678136 & -1.282548 & 3.094347 \\ \mathrm{H} & -0.046508 & 0.269103 & 3.713391 \\ \mathrm{H} & 0.979609 & -0.658945 & 2.632455 \\ \mathrm{H} & 1.189878 & 1.480581 & 1.277914 \\ \mathrm{O} & -5.069455 & -2.537054 & -0.625578 \\ \mathrm{~S} & -3.169697 & -0.906072 & 1.912608\end{array}$

TS2 Cs transition state

$\begin{array}{lrrr}\mathrm{H} & 1.659217 & -3.280746 & 0.385123 \\ \mathrm{C} & 1.709170 & -2.761704 & -0.584131 \\ \mathrm{O} & 0.461714 & -2.953624 & -1.272466 \\ \mathrm{C} & 0.047510 & -1.739650 & -1.898339 \\ \mathrm{C} & 1.894348 & -1.250457 & -0.440602 \\ \mathrm{C} & 1.176616 & -0.725107 & -1.683716 \\ \mathrm{O} & 3.263084 & -0.904545 & -0.376530 \\ \mathrm{H} & 2.546566 & -3.176605 & -1.174167 \\ \mathrm{H} & -0.141785 & -1.913003 & -2.965101 \\ \mathrm{H} & 1.377498 & -0.904798 & 0.464591 \\ \mathrm{H} & 0.822509 & 0.307869 & -1.585947 \\ \mathrm{H} & 1.872000 & -0.773876 & -2.537529 \\ \mathrm{~N} & -1.276125 & -1.371879 & -1.351464 \\ \mathrm{C} & -2.278228 & -1.094437 & -2.253217 \\ \mathrm{O} & -2.096559 & -0.806363 & -3.428192 \\ \mathrm{~N} & -3.579792 & -1.185433 & -1.721165 \\ \mathrm{C} & -3.944592 & -2.046713 & -0.663689 \\ \mathrm{C} & -2.886944 & -2.251164 & 0.353882 \\ \mathrm{C} & -2.810811 & -3.674185 & 0.856905 \\ \mathrm{C} & -1.580358 & -1.527456 & 0.046973 \\ \mathrm{H} & -4.297541 & -1.075289 & -2.433801 \\ \mathrm{H} & -2.320315 & -4.312650 & 0.099602\end{array}$




$\begin{array}{cccc}\mathrm{H} & -3.824811 & -4.060787 & 1.032838 \\ \mathrm{H} & -2.230006 & -3.730813 & 1.789326 \\ \mathrm{H} & -0.752392 & -2.064247 & 0.517969 \\ \mathrm{P} & 3.794875 & -0.216043 & 1.087884 \\ \mathrm{O} & 2.936097 & -0.784603 & 2.207063 \\ \mathrm{O} & 5.298175 & -0.141864 & 1.019040 \\ \mathrm{O} & 3.156298 & 1.352637 & 0.867281 \\ \mathrm{C} & 3.701733 & 2.153835 & -0.159305 \\ \mathrm{C} & 2.740592 & 3.301532 & -0.423283 \\ \mathrm{O} & 1.543550 & 2.784084 & -1.051455 \\ \mathrm{C} & 0.370630 & 3.318140 & -0.474231 \\ \mathrm{C} & 2.268203 & 4.033596 & 0.840715 \\ \mathrm{C} & 0.822186 & 4.415020 & 0.507780 \\ \mathrm{H} & 2.903212 & 4.902708 & 1.069234 \\ \mathrm{H} & 3.836999 & 1.570370 & -1.086229 \\ \mathrm{H} & 4.689313 & 2.555576 & 0.137327 \\ \mathrm{H} & 3.210027 & 4.007513 & -1.136476 \\ \mathrm{H} & -0.288888 & 3.704579 & -1.258257 \\ \mathrm{H} & 2.312592 & 3.340508 & 1.692062 \\ \mathrm{H} & 0.163662 & 4.476871 & 1.385208 \\ \mathrm{H} & 0.784225 & 5.383002 & -0.018871 \\ \mathrm{~N} & -0.423429 & 2.237216 & 0.146172 \\ \mathrm{C} & -1.721993 & 2.030361 & -0.380099 \\ \mathrm{O} & -2.237554 & 2.818918 & -1.165802 \\ \mathrm{~N} & -2.350792 & 0.905000 & 0.056882 \\ \mathrm{C} & -1.821659 & -0.153266 & 0.891338 \\ \mathrm{C} & -0.674217 & 0.332845 & 1.674564 \\ \mathrm{C} & -0.091704 & -0.393610 & 2.828729 \\ \mathrm{C} & 0.108859 & 1.431159 & 1.115453 \\ \mathrm{H} & -3.246352 & 0.737801 & -0.386819 \\ \mathrm{H} & -0.683192 & -1.279381 & 3.095271 \\ \mathrm{H} & -0.054128 & 0.273281 & 3.714372 \\ \mathrm{H} & 0.975093 & -0.654852 & 2.636470 \\ \mathrm{H} & 1.187044 & 1.482267 & 1.279122 \\ \mathrm{O} & -5.066405 & -2.539638 & -0.632986 \\ \mathrm{~S} & -3.172571 & -0.905914 & 1.908350\end{array}$

I2 minimum
H 1.619999
$-3.602008$
$-0.278955$
C 1.633525
$-2.826067$
$-1.059279$
$\begin{array}{llll}\text { O } & 0.313904 & -2.738783 & -1.624439\end{array}$
$\begin{array}{llll}\text { C } & 0.020291 & -1.375441 & -1.966609\end{array}$
$\begin{array}{llll}\text { C } & 1.960187 & -1.430577 & -0.523592\end{array}$
$\begin{array}{llll}\text { C } & 1.224855 & -0.543614 & -1.537687\end{array}$
$\begin{array}{llll}\text { O } & 3.350655 & -1.208541 & -0.493367\end{array}$
H $\quad 2.371285 \quad-3.104121 \quad-1.834459$
$\mathrm{H} \quad-0.180820 \quad-1.290134 \quad-3.041769$
$\begin{array}{llll}\mathrm{H} & 1.546953 & -1.314265 & 0.493217\end{array}$ 


\begin{tabular}{|c|c|c|c|}
\hline $\mathrm{H}$ & .956901 & & \\
\hline & 1.895707 & -0.409518 & -2.400939 \\
\hline & -1.266742 & & \\
\hline C & -2.345144 & -0.884989 & -2.174011 \\
\hline & -2.291046 & -0.586249 & -3.353777 \\
\hline & -3.608059 & -1.142248 & -1.57510 \\
\hline $\mathrm{C}$ & -3.789418 & -2.140917 & -0. \\
\hline $\mathrm{C}$ & -2.646697 & -2.266754 & \\
\hline $\mathrm{C}$ & -2.284878 & -3.718 & \\
\hline $\mathrm{C}$ & -1.471342 & -1.3 & \\
\hline $\mathrm{H}$ & -4.360920 & & \\
\hline $\mathrm{H}$ & -1.760249 & -4.104274 & -0.2 \\
\hline[ & -3.190824 & -4.3 & \\
\hline $\mathrm{H}$ & -1.609378 & & \\
\hline $\mathrm{H}$ & -0.54 & -1.7 & \\
\hline$P$ & 3.935322 & -0.38 & \\
\hline $\mathrm{O}$ & 3.232303 & & \\
\hline $\mathrm{O}$ & 5.41 & & \\
\hline $\mathrm{O}$ & 3.13 & & \\
\hline $\mathrm{C}$ & 3.65 & & \\
\hline C & 2.69 & & \\
\hline $\mathrm{O}$ & 1.45 & & \\
\hline $\mathrm{C}$ & 0.32 & & \\
\hline $\mathrm{C}$ & 2.31 & & \\
\hline $\mathrm{C}$ & 0.87 & & \\
\hline $\mathrm{H}$ & 2.99 & & \\
\hline $\mathrm{H}$ & 3.75 & & \\
\hline $\mathrm{H}$ & 4.65 & & -0. \\
\hline $\mathrm{H}$ & 3.15 & & -1 . \\
\hline $\mathrm{H}$ & -0.35 & & \\
\hline $\mathrm{H}$ & 2.37 & & \\
\hline $\mathrm{H}$ & 0.26 & & \\
\hline $\mathrm{H}$ & 0.84 & & \\
\hline $\mathrm{N}$ & -0.46 & & \\
\hline $\mathrm{C}$ & -1.799950 & & \\
\hline $\mathrm{O}$ & -2.34 & & \\
\hline $\mathrm{N}$ & -2.510548 & 439 & \\
\hline $\mathrm{C}$ & -1.880093 & -0.12 & \\
\hline $\mathrm{C}$ & -0.79 & & \\
\hline $\mathrm{C}$ & -1.07 & 0.7 & \\
\hline $\mathrm{C}$ & 0.123888 & 1.272301 & \\
\hline $\mathrm{H}$ & -3.367323 & & 7009 \\
\hline $\mathrm{H}$ & -1.74 & & \\
\hline $\mathrm{H}$ & -0.129176 & 1.015321 & \\
\hline $\mathrm{H}$ & -1.561021 & & 3.857896 \\
\hline $\mathrm{H}$ & 1.203178 & 1.114774 & 1.047039 \\
\hline $\mathrm{O}$ & -4.799306 & & \\
\hline & -3.151153 & -1.236475 & 1.866557 \\
\hline
\end{tabular}


TS3 transition state

$\begin{array}{lccc}\mathrm{H} & 1.813051 & -3.703475 & -0.355517 \\ \mathrm{C} & 1.767084 & -2.837065 & -1.031594 \\ \mathrm{O} & 0.449786 & -2.788750 & -1.596058 \\ \mathrm{C} & 0.105820 & -1.423061 & -1.887426 \\ \mathrm{C} & 1.957301 & -1.495281 & -0.323774 \\ \mathrm{C} & 1.207210 & -0.551545 & -1.275831 \\ \mathrm{O} & 3.323393 & -1.187823 & -0.165758 \\ \mathrm{H} & 2.533659 & -2.953647 & -1.821753 \\ \mathrm{H} & 0.009029 & -1.278221 & -2.971204 \\ \mathrm{H} & 1.473432 & -1.524798 & 0.666570 \\ \mathrm{H} & 0.828740 & 0.359154 & -0.805956 \\ \mathrm{H} & 1.910128 & -0.241692 & -2.063531 \\ \mathrm{~N} & -1.243393 & -1.194873 & -1.363583 \\ \mathrm{C} & -2.274719 & -1.049837 & -2.257929 \\ \mathrm{O} & -2.163305 & -0.808185 & -3.446631 \\ \mathrm{~N} & -3.569457 & -1.224274 & -1.698535 \\ \mathrm{C} & -3.848330 & -2.115618 & -0.661343 \\ \mathrm{C} & -2.747848 & -2.229912 & 0.378682 \\ \mathrm{C} & -2.483260 & -3.682954 & 0.733063 \\ \mathrm{C} & -1.505299 & -1.383635 & 0.042953 \\ \mathrm{H} & -4.294417 & -1.173275 & -2.411698 \\ \mathrm{H} & -1.990905 & -4.177769 & -0.121625 \\ \mathrm{H} & -3.427629 & -4.203412 & 0.947518 \\ \mathrm{H} & -1.815082 & -3.747888 & 1.604260 \\ \mathrm{H} & -0.626970 & -1.863794 & 0.482275 \\ \mathrm{P} & 3.733708 & -0.246903 & 1.195902 \\ \mathrm{O} & 2.878848 & -0.704305 & 2.366321 \\ \mathrm{O} & 5.230828 & -0.073619 & 1.165972 \\ \mathrm{O} & 2.997212 & 1.213375 & 0.724588 \\ \mathrm{C} & 3.601694 & 1.997003 & -0.277891 \\ \mathrm{C} & 2.707858 & 3.203599 & -0.523149 \\ \mathrm{O} & 1.458108 & 2.768067 & -1.100023 \\ \mathrm{C} & 0.336585 & 3.363194 & -0.465561 \\ \mathrm{C} & 2.340497 & 3.978859 & 0.751214 \\ \mathrm{C} & 0.895329 & 4.426993 & 0.495949 \\ \mathrm{H} & 3.027487 & 4.818659 & 0.931350 \\ \mathrm{H} & 3.716325 & 1.423926 & -1.216288 \\ \mathrm{H} & 4.609443 & 2.328095 & 0.036970 \\ \mathrm{H} & 3.205041 & 3.863303 & -1.262114 \\ \mathrm{H} & -0.318931 & 3.787333 & -1.232521 \\ & 2.396115 & 3.297458 & 1.610500 \\ \mathrm{H} & 0.295167 & 4.498443 & 1.413525 \\ \mathrm{H} & -0.493374 & 2.340744 & 0.191921 \\ \mathrm{H} & -2.731988 & 2.066326 & -0.392904 \\ & 0.997170 & 0.143583\end{array}$




$\begin{array}{lrrl}\mathrm{C} & -1.830401 & -0.076289 & 0.902242 \\ \mathrm{C} & -0.778335 & 0.456974 & 1.780060 \\ \mathrm{C} & -0.189537 & -0.269034 & 2.928367 \\ \mathrm{C} & -0.021207 & 1.680135 & 1.320910 \\ \mathrm{H} & -3.237387 & 0.760781 & -0.419788 \\ \mathrm{H} & -0.159822 & 0.406339 & 3.809873 \\ \mathrm{H} & 0.880173 & -0.524463 & 2.744034 \\ \mathrm{H} & -0.762186 & -1.167276 & 3.196051 \\ \mathrm{H} & 1.068335 & 1.602651 & 1.375145 \\ \mathrm{O} & -4.902683 & -2.720511 & -0.608246 \\ \mathrm{~S} & -3.198683 & -1.087384 & 1.798013\end{array}$

I3 minimum

$\begin{array}{llll}\mathrm{H} & 1.756147 & -3.235030 & 1.262673 \\ \mathrm{C} & 1.791905 & -3.042675 & 0.179922 \\ \mathrm{O} & 0.545847 & -3.484501 & -0.388946 \\ \mathrm{C} & 0.163776 & -2.613399 & -1.454460 \\ \mathrm{C} & 1.915844 & -1.562350 & -0.173541 \\ \mathrm{C} & 1.243144 & -1.527674 & -1.549500 \\ \mathrm{O} & 3.260230 & -1.143381 & -0.180135 \\ \mathrm{H} & 2.640375 & -3.599050 & -0.260937 \\ \mathrm{H} & 0.063457 & -3.182345 & -2.387735 \\ \mathrm{H} & 1.340897 & -0.962832 & 0.539244 \\ \mathrm{H} & 0.842123 & -0.541393 & -1.811510 \\ \mathrm{H} & 1.992017 & -1.821633 & -2.301994 \\ \mathrm{~N} & -1.198056 & -2.119833 & -1.174152 \\ \mathrm{C} & -2.071806 & -2.006571 & -2.212291 \\ \mathrm{O} & -1.837882 & -2.271872 & -3.382587 \\ \mathrm{~N} & -3.358238 & -1.527595 & -1.846819 \\ \mathrm{C} & -3.968056 & -1.668087 & -0.604287 \\ \mathrm{C} & -2.997724 & -2.048295 & 0.514959 \\ \mathrm{C} & -3.096393 & -3.571042 & 0.708662 \\ \mathrm{C} & -1.534984 & -1.642389 & 0.175315 \\ \mathrm{H} & -3.988933 & -1.436538 & -2.640136 \\ \mathrm{H} & -2.756387 & -4.090470 & -0.201667 \\ \mathrm{H} & -4.133624 & -3.867124 & 0.920387 \\ \mathrm{H} & -2.450111 & -3.882265 & 1.541991 \\ \mathrm{H} & -0.903694 & -2.230153 & 0.846289 \\ \mathrm{P} & 3.597383 & 0.347445 & 0.554370 \\ \mathrm{O} & 2.923331 & 0.388886 & 1.924399 \\ \mathrm{O} & 5.057646 & 0.640965 & 0.306536 \\ \mathrm{O} & 2.629873 & 1.321492 & -0.459889 \\ \mathrm{C} & 3.186808 & 2.530736 & -0.939866 \\ \mathrm{C} & 2.474901 & 3.745108 & -0.357786 \\ \mathrm{O} & 1.110736 & 3.804387 & -0.839713 \\ \mathrm{C} & 0.187754 & 3.819138 & 0.229092 \\ \mathrm{C} & 2.393748 & 3.781821 & 1.178528\end{array}$




$\begin{array}{lrrr}\mathrm{C} & 0.978840 & 4.325438 & 1.439515 \\ \mathrm{H} & 3.175103 & 4.423085 & 1.609974 \\ \mathrm{H} & 3.075579 & 2.562151 & -2.039045 \\ \mathrm{H} & 4.262631 & 2.578096 & -0.695436 \\ \mathrm{H} & 2.980807 & 4.648882 & -0.753229 \\ \mathrm{H} & -0.635394 & 4.476804 & -0.073495 \\ \mathrm{H} & 2.520802 & 2.767108 & 1.586666 \\ \mathrm{H} & 0.545176 & 4.023004 & 2.402569 \\ \mathrm{H} & 0.968184 & 5.426431 & 1.405349 \\ \mathrm{~N} & -0.421560 & 2.489565 & 0.428388 \\ \mathrm{C} & -1.311802 & 2.086106 & -0.572221 \\ \mathrm{O} & -1.778002 & 2.854571 & -1.402326 \\ \mathrm{~N} & -1.629209 & 0.743304 & -0.556603 \\ \mathrm{C} & -1.209139 & -0.197128 & 0.406616 \\ \mathrm{C} & -0.442851 & 0.282969 & 1.474291 \\ \mathrm{C} & 0.066877 & -0.553606 & 2.623050 \\ \mathrm{C} & -0.040213 & 1.614407 & 1.444523 \\ \mathrm{H} & -2.312900 & 0.483006 & -1.257986 \\ \mathrm{H} & -0.424854 & -0.246968 & 3.560591 \\ \mathrm{H} & 1.155337 & -0.394722 & 2.710475 \\ \mathrm{H} & -0.139127 & -1.624003 & 2.493621 \\ \mathrm{H} & 0.665762 & 1.982803 & 2.176620 \\ \mathrm{O} & -5.168139 & -1.540221 & -0.467916 \\ \mathrm{~S} & -3.426049 & -1.165864 & 2.068444\end{array}$

TS4 transition state

$\begin{array}{llll}\mathrm{H} & 1.997672 & -3.388624 & 0.998897 \\ \mathrm{C} & 1.898694 & -3.011000 & -0.028725 \\ \mathrm{O} & 0.575758 & -3.319648 & -0.484710 \\ \mathrm{C} & 0.206063 & -2.373286 & -1.499863 \\ \mathrm{C} & 2.013093 & -1.495683 & -0.141837 \\ \mathrm{C} & 1.231164 & -1.224420 & -1.435767 \\ \mathrm{O} & 3.358008 & -1.078255 & -0.162649 \\ \mathrm{H} & 2.663480 & -3.496267 & -0.666371 \\ \mathrm{H} & 0.214480 & -2.860599 & -2.482547 \\ \mathrm{H} & 1.501457 & -1.032549 & 0.704249 \\ \mathrm{H} & 0.777522 & -0.227554 & -1.460670 \\ \mathrm{H} & 1.930732 & -1.305720 & -2.281874 \\ \mathrm{~N} & -1.211169 & -2.026126 & -1.287675 \\ \mathrm{C} & -2.059393 & -2.244641 & -2.336597 \\ \mathrm{O} & -1.718454 & -2.515992 & -3.479705 \\ \mathrm{~N} & -3.436655 & -2.160160 & -2.037392 \\ \mathrm{C} & -3.981471 & -2.411204 & -0.791639 \\ \mathrm{C} & -3.152883 & -1.905884 & 0.382700 \\ \mathrm{C} & -3.269581 & -2.861491 & 1.570821 \\ \mathrm{C} & -1.627756 & -1.634396 & 0.080680 \\ \mathrm{H} & -4.011895 & -2.455038 & -2.822996 \\ \mathrm{H} & -2.774896 & -3.816442 & 1.320070\end{array}$




$\begin{array}{cccc}\mathrm{H} & -4.322765 & -3.067564 & 1.803114 \\ \mathrm{H} & -2.774376 & -2.422426 & 2.448726 \\ \mathrm{H} & -1.074829 & -2.313947 & 0.734973 \\ \mathrm{P} & 3.679258 & 0.369722 & 0.653460 \\ \mathrm{O} & 2.968989 & 0.337359 & 2.006762 \\ \mathrm{O} & 5.143344 & 0.682812 & 0.458909 \\ \mathrm{O} & 2.731944 & 1.386881 & -0.337517 \\ \mathrm{C} & 3.271851 & 2.639588 & -0.709300 \\ \mathrm{C} & 2.533946 & 3.788022 & -0.033759 \\ \mathrm{O} & 1.171408 & 3.852982 & -0.516352 \\ \mathrm{C} & 0.242697 & 3.765950 & 0.543902 \\ \mathrm{C} & 2.448983 & 3.696890 & 1.500308 \\ \mathrm{C} & 1.018771 & 4.174329 & 1.801556 \\ \mathrm{H} & 3.210120 & 4.325316 & 1.983723 \\ \mathrm{H} & 3.164990 & 2.758800 & -1.802791 \\ \mathrm{H} & 4.345489 & 2.684055 & -0.454435 \\ \mathrm{H} & 3.020990 & 4.731851 & -0.351575 \\ \mathrm{H} & -0.583566 & 4.443064 & 0.297138 \\ \mathrm{H} & 2.605513 & 2.658204 & 1.829709 \\ \mathrm{H} & 0.590970 & 3.765646 & 2.727193 \\ \mathrm{H} & 0.977198 & 5.272502 & 1.875081 \\ \mathrm{~N} & -0.362305 & 2.422092 & 0.623859 \\ \mathrm{C} & -1.254866 & 2.114542 & -0.396034 \\ \mathrm{O} & -1.694922 & 2.932651 & -1.191507 \\ \mathrm{~N} & -1.624286 & 0.776053 & -0.456512 \\ \mathrm{C} & -1.184433 & -0.247244 & 0.416590 \\ \mathrm{C} & -0.374318 & 0.134911 & 1.483296 \\ \mathrm{C} & 0.084548 & -0.797735 & 2.579803 \\ \mathrm{C} & 0.058560 & 1.448362 & 1.540449 \\ \mathrm{H} & -2.162672 & 0.544638 & -1.283294 \\ \mathrm{H} & -0.512606 & -0.645090 & 3.495721 \\ \mathrm{H} & 1.146105 & -0.589864 & 2.793750 \\ \mathrm{H} & -0.009471 & -1.856596 & 2.299316 \\ \mathrm{H} & 0.805353 & 1.742154 & 2.265059 \\ \mathrm{O} & -5.077996 & -2.926941 & -0.661656 \\ \mathrm{~S} & -4.004445 & -0.292837 & 0.793757\end{array}$

I4 minimum

$\begin{array}{llll}\mathrm{H} & 2.121639 & -3.532793 & 0.529874 \\ \mathrm{C} & 1.989420 & -2.964532 & -0.402161 \\ \mathrm{O} & 0.679159 & -3.242106 & -0.905032 \\ \mathrm{C} & 0.231853 & -2.126432 & -1.705048 \\ \mathrm{C} & 2.016288 & -1.452269 & -0.206997 \\ \mathrm{C} & 1.220193 & -0.981066 & -1.432135 \\ \mathrm{O} & 3.329116 & -0.941916 & -0.138986 \\ \mathrm{H} & 2.773124 & -3.268875 & -1.124185 \\ \mathrm{H} & 0.242517 & -2.418601 & -2.760411 \\ \mathrm{H} & 1.481415 & -1.203146 & 0.714794\end{array}$




\begin{tabular}{|c|c|c|c|}
\hline & 0.774395 & 0.004968 & \\
\hline & 1.927679 & -0.916142 & -2.273716 \\
\hline & -1.210005 & -1.939614 & .415968 \\
\hline & -1.988898 & & -2.135741 \\
\hline & -1.709282 & -3.264402 & -3.246304 \\
\hline & -3.149745 & -3.322720 & -1.512426 \\
\hline & -3.746014 & -2.901864 & -0.346464 \\
\hline & -3.118288 & -1.647383 & 0.247522 \\
\hline & -3.397889 & -1.576694 & 1.747584 \\
\hline & -1.580320 & & -0.018873 \\
\hline & -3.6298 & & -2.0 \\
\hline & -2.879934 & -2.3 & 2.277096 \\
\hline & -4.47 & -1. & \\
\hline & -3.0 & & \\
\hline & -1.12 & & \\
\hline & 3.520656 & 0.4 & 0.812888 \\
\hline & 2.77 & & \\
\hline & 4.96 & & \\
\hline & 2.54 & & \\
\hline & 3.09 & & \\
\hline & 2.43 & & \\
\hline & 1.07 & & \\
\hline & 0.151923 & & \\
\hline & 2.356371 & & \\
\hline & 0.91 & & \\
\hline & 3.10 & & \\
\hline & 2.89 & & \\
\hline & 4.18 & & \\
\hline & 2.96 & & \\
\hline & -0.70 & & \\
\hline & 2.522936 & & \\
\hline & 0.50 & & \\
\hline & 0.85 & & \\
\hline & -0.3714 & & \\
\hline & -1.09 & & -0.4 \\
\hline & -1.45 & & -1.2 \\
\hline N & -1.391831 & & -0.573998 \\
\hline & -1.060774 & & 0.342561 \\
\hline & -0.401096 & & \\
\hline C & -0.031770 & $-0 . \varepsilon$ & \\
\hline $\mathrm{C}$ & -0.023049 & & \\
\hline $\mathrm{H}$ & -1.676719 & & \\
\hline & -0.69 & & \\
\hline $\mathrm{H}$ & & & \\
\hline $\mathrm{H}$ & -0.105859 & & 2.292759 \\
\hline $\mathrm{H}$ & 0.632848 & & \\
\hline $\mathrm{O}$ & -4.715256 & & \\
\hline & -4.003593 & -0.272358 & -0.643704 \\
\hline
\end{tabular}


TS5 transition state

\begin{tabular}{|c|c|c|c|}
\hline & 2.226710 & -3.292207 & \\
\hline$c$ & 2.051211 & -2.884021 & \\
\hline C & 0.745862 & -3.275707 & 9506 \\
\hline & 0.251407 & -2.314222 & \\
\hline & .023281 & -1.361043 & -0.224621 \\
\hline C & .201901 & & \\
\hline & 3.318758 & & 2056 \\
\hline & 2.836050 & -3.267063 & \\
\hline & 0.277129 & & 902 \\
\hline & & & \\
\hline & 0.697344 & -0.13 & 0173 \\
\hline & 1.893763 & -1.13 & \\
\hline & 57 & -2 & -1 . \\
\hline & -1.936 & & \\
\hline & -1.612181 & -3.7 & 3729 \\
\hline & -3.120872 & -3.52 & -1.268797 \\
\hline & & & -0.2 \\
\hline & -3.1 & -1.5 & \\
\hline & -3.6 & -1.1 & \\
\hline & -1.6 & -1.6 & \\
\hline F & -3.5 & & \\
\hline & -3.17 & -1.7 & \\
\hline & -4.71 & -1.2 & 1. \\
\hline & -3.32 & -0.0 & \\
\hline & -1.3 & -2.3 & \\
\hline & 3.50 & 0.5 & \\
\hline & 2.74 & 0.4 & 2.0 \\
\hline $\mathrm{O}$ & 4.94 & & \\
\hline $\mathrm{O}$ & 2.54 & & -0.2 \\
\hline $\mathrm{C}$ & 3.057372 & 2.8 & -0.6 \\
\hline $\mathrm{C}$ & 2.380605 & 4.00 & \\
\hline $\mathrm{O}$ & 0.99 & & \\
\hline $\mathrm{C}$ & 0.13 & & \\
\hline $\mathrm{C}$ & & & \\
\hline $\mathrm{C}$ & 0.92 & & \\
\hline $\mathrm{H}$ & 3.10 & & \\
\hline $\mathrm{H}$ & & & \\
\hline $\mathrm{H}$ & & 2.92 & \\
\hline $\mathrm{H}$ & & & \\
\hline & -0.774349 & & \\
\hline & & 2.76 & \\
\hline & & & \\
\hline & 0.80 & 5.23 & \\
\hline $\mathrm{N}$ & -0.295049 & & 0.771764 \\
\hline & -1.070283 & 2.038212 & -0.356733 \\
\hline & & & \\
\hline $\mathrm{N}$ & & 0.703933 & -0.617693 \\
\hline & -1.044192 & -0.275343 & 0.32926 \\
\hline
\end{tabular}




$\begin{array}{lrrr}\mathrm{C} & -0.358451 & 0.063130 & 1.499900 \\ \mathrm{C} & 0.010056 & -0.897609 & 2.588805 \\ \mathrm{C} & 0.051197 & 1.412130 & 1.690118 \\ \mathrm{H} & -2.578391 & 0.353437 & -1.221150 \\ \mathrm{H} & -0.628881 & -0.716690 & 3.472602 \\ \mathrm{H} & 1.061292 & -0.707593 & 2.863652 \\ \mathrm{H} & -0.105076 & -1.946916 & 2.291279 \\ \mathrm{H} & 0.751123 & 1.659547 & 2.474630 \\ \mathrm{O} & -4.811474 & -3.346707 & 0.236734 \\ \mathrm{~S} & -3.905934 & -0.348076 & -1.148147\end{array}$

I5 minimum

$\begin{array}{lccc}\mathrm{H} & 2.166591 & -3.424728 & 0.536123 \\ \mathrm{C} & 2.014907 & -2.902973 & -0.420496 \\ \mathrm{O} & 0.714480 & -3.227037 & -0.909538 \\ \mathrm{C} & 0.223135 & -2.139211 & -1.730843 \\ \mathrm{C} & 2.013428 & -1.384177 & -0.285235 \\ \mathrm{C} & 1.205916 & -0.970957 & -1.523022 \\ \mathrm{O} & 3.323896 & -0.861830 & -0.204310 \\ \mathrm{H} & 2.809066 & -3.215188 & -1.128407 \\ \mathrm{H} & 0.215024 & -2.464114 & -2.776551 \\ \mathrm{H} & 1.461296 & -1.117964 & 0.622761 \\ \mathrm{H} & 0.727449 & 0.006031 & -1.437639 \\ \mathrm{H} & 1.894139 & -0.935145 & -2.381937 \\ \mathrm{~N} & -1.209738 & -1.979592 & -1.413479 \\ \mathrm{C} & -1.974902 & -2.930232 & -2.082360 \\ \mathrm{O} & -1.713374 & -3.377216 & -3.188132 \\ \mathrm{~N} & -3.088519 & -3.445163 & -1.403107 \\ \mathrm{C} & -3.715592 & -2.946695 & -0.284364 \\ \mathrm{C} & -3.134199 & -1.630458 & 0.201309 \\ \mathrm{C} & -3.441666 & -1.428479 & 1.683627 \\ \mathrm{C} & -1.592339 & -1.574857 & -0.041400 \\ \mathrm{H} & -3.569968 & -4.184927 & -1.905943 \\ \mathrm{H} & -2.881609 & -2.151871 & 2.300134 \\ \mathrm{H} & -4.512619 & -1.589167 & 1.859440 \\ \mathrm{H} & -3.169119 & -0.411812 & 1.994711 \\ \mathrm{H} & -1.158140 & -2.304787 & 0.659364 \\ \mathrm{P} & 3.522067 & 0.429786 & 0.872700 \\ \mathrm{O} & 2.765004 & 0.113468 & 2.164081 \\ \mathrm{O} & 4.960058 & 0.884238 & 0.814669 \\ \mathrm{O} & 2.533528 & 1.549346 & 0.054064 \\ \mathrm{C} & 3.106186 & 2.719372 & -0.496453 \\ \mathrm{C} & 2.465372 & 3.955628 & 0.122020 \\ \mathrm{O} & 1.095255 & 4.089444 & -0.328346 \\ \mathrm{C} & 0.193337 & 3.850266 & 0.729636 \\ \mathrm{C} & 2.418899 & 3.942931 & 1.667126 \\ \mathrm{C} & 0.947450 & 4.258002 & 1.997170 \\ \mathrm{H} & 3.100229 & 4.690601 & 2.096181\end{array}$




$\begin{array}{lrrr}\mathrm{H} & 2.913438 & 2.739841 & -1.584985 \\ \mathrm{H} & 4.195855 & 2.727798 & -0.319632 \\ \mathrm{H} & 2.993620 & 4.846116 & -0.264375 \\ \mathrm{H} & -0.688395 & 4.468998 & 0.526515 \\ \mathrm{H} & 2.712106 & 2.956057 & 2.051286 \\ \mathrm{H} & 0.578945 & 3.766712 & 2.907400 \\ \mathrm{H} & 0.802098 & 5.342187 & 2.121518 \\ \mathrm{~N} & -0.290577 & 2.454475 & 0.723671 \\ \mathrm{C} & -1.098320 & 2.095524 & -0.378087 \\ \mathrm{O} & -1.574620 & 2.973549 & -1.094122 \\ \mathrm{~N} & -1.354159 & 0.769254 & -0.643353 \\ \mathrm{C} & -1.061635 & -0.195555 & 0.292055 \\ \mathrm{C} & -0.384270 & 0.137852 & 1.467087 \\ \mathrm{C} & -0.054211 & -0.804937 & 2.573507 \\ \mathrm{C} & 0.068411 & 1.492678 & 1.649704 \\ \mathrm{H} & -2.959547 & 0.333333 & -1.175965 \\ \mathrm{H} & -0.562220 & -0.474378 & 3.496939 \\ \mathrm{H} & 1.038189 & -0.730620 & 2.746699 \\ \mathrm{H} & -0.342164 & -1.839841 & 2.363479 \\ \mathrm{H} & 0.895782 & 1.680812 & 2.323864 \\ \mathrm{O} & -4.686777 & -3.495509 & 0.208253 \\ \mathrm{~S} & -4.105400 & -0.379850 & -0.824520\end{array}$

P1 transition state

$\begin{array}{lrrr}\mathrm{H} & 1.968932 & -2.885675 & 1.010004 \\ \mathrm{C} & 1.848379 & -2.743714 & -0.071066 \\ \mathrm{O} & 0.574778 & -3.268983 & -0.479805 \\ \mathrm{C} & 0.045287 & -2.476178 & -1.539510 \\ \mathrm{C} & 1.841027 & -1.270024 & -0.477935 \\ \mathrm{C} & 1.036313 & -1.313843 & -1.776085 \\ \mathrm{O} & 3.142154 & -0.737249 & -0.617823 \\ \mathrm{H} & 2.671768 & -3.259847 & -0.600717 \\ \mathrm{H} & -0.077398 & -3.082881 & -2.446791 \\ \mathrm{H} & 1.278507 & -0.692477 & 0.264341 \\ \mathrm{H} & 0.518341 & -0.375622 & -2.012462 \\ \mathrm{H} & 1.711888 & -1.566610 & -2.608775 \\ \mathrm{~N} & -1.326875 & -2.094735 & -1.157181 \\ \mathrm{C} & -2.222652 & -1.899279 & -2.170959 \\ \mathrm{O} & -1.976185 & -2.028877 & -3.362453 \\ \mathrm{~N} & -3.539758 & -1.587572 & -1.769212 \\ \mathrm{C} & -4.003525 & -1.390156 & -0.494370 \\ \mathrm{C} & -3.057185 & -1.947489 & 0.579412 \\ \mathrm{C} & -3.260280 & -3.472745 & 0.642784 \\ \mathrm{C} & -1.588832 & -1.604015 & 0.207692 \\ \mathrm{H} & -4.130832 & -1.254855 & -2.525053 \\ \mathrm{H} & -3.001782 & -3.947458 & -0.315614 \\ \mathrm{H} & -4.304813 & -3.704697 & 0.895048 \\ \mathrm{H} & -2.610794 & -3.900558 & 1.420403\end{array}$




$\begin{array}{lccc}\mathrm{H} & -0.942072 & -2.201416 & 0.857640 \\ \mathrm{P} & 3.784074 & -0.063772 & 0.810185 \\ \mathrm{O} & 3.126724 & -0.757004 & 2.003176 \\ \mathrm{O} & 5.265881 & 0.107719 & 0.590906 \\ \mathrm{O} & 3.021813 & 1.451714 & 0.737992 \\ \mathrm{C} & 3.566725 & 2.373688 & -0.188343 \\ \mathrm{C} & 2.664987 & 3.594585 & -0.285619 \\ \mathrm{O} & 1.461212 & 3.260335 & -1.004119 \\ \mathrm{C} & 0.291929 & 3.669610 & -0.311040 \\ \mathrm{C} & 2.219067 & 4.175328 & 1.068029 \\ \mathrm{C} & 0.762529 & 4.594914 & 0.821979 \\ \mathrm{H} & 2.854436 & 5.016183 & 1.381062 \\ \mathrm{H} & 3.655005 & 1.917592 & -1.189888 \\ \mathrm{H} & 4.579337 & 2.683674 & 0.130468 \\ \mathrm{H} & 3.199419 & 4.358485 & -0.884837 \\ \mathrm{H} & -0.373038 & 4.167288 & -1.023976 \\ \mathrm{H} & 2.294405 & 3.389664 & 1.832216 \\ \mathrm{H} & 0.118659 & 4.520159 & 1.710306 \\ \mathrm{H} & 0.712368 & 5.633632 & 0.458977 \\ \mathrm{~N} & -0.459591 & 2.500111 & 0.140680 \\ \mathrm{C} & -1.425415 & 1.940529 & -0.768543 \\ \mathrm{O} & -1.863084 & 2.670682 & -1.660342 \\ \mathrm{~N} & -1.814589 & 0.648567 & -0.626824 \\ \mathrm{C} & -1.248285 & -0.140324 & 0.332838 \\ \mathrm{C} & -0.430277 & 0.392624 & 1.344278 \\ \mathrm{C} & 0.116113 & -0.355291 & 2.504292 \\ \mathrm{C} & -0.067759 & 1.769348 & 1.242692 \\ \mathrm{H} & -4.690248 & -0.774322 & 1.768724 \\ \mathrm{H} & -0.323278 & -1.352786 & 2.624461 \\ \mathrm{H} & -0.057889 & 0.221569 & 3.428569 \\ \mathrm{H} & 1.229587 & -0.457079 & 2.396379 \\ \mathrm{H} & 0.656806 & 2.204646 & 1.920355 \\ \mathrm{O} & -5.109127 & -0.919207 & -0.274296 \\ \mathrm{~S} & -3.478273 & -1.200167 & 2.222220\end{array}$

